\title{
Effect of neutron alignments on the structure of ${ }^{197} \mathrm{Tl}$
}

\author{
S. Nandi, ${ }^{1,2}$ G. Mukherjee, ${ }^{1,2, *}$ T. Roy, ${ }^{1,2}$ R. Banik, ${ }^{1,2}$ A. Dhal,${ }^{1}$ Soumik Bhattacharya, ${ }^{1,2}$ S. Bhattacharyya,${ }^{1,2}$ \\ C. Bhattacharya, ${ }^{1,2}$ Md. A. Asgar, ${ }^{1,2,3}$ H. Pai, ${ }^{4}$ S. Rajbanshi, ${ }^{5}$ Pratap Roy, ${ }^{1}$ T. K. Ghosh, ${ }^{1,2}$ K. Banerjee, ${ }^{1,2,6}$ T. K. Rana, ${ }^{1}$ \\ Samir Kundu, ${ }^{1}$ S. Manna, ${ }^{1,2}$ R. Pandey, ${ }^{1}$ A. Sen, ${ }^{1}$ S. Pal, ${ }^{1,2}$ S. Mukhopadhyay, ${ }^{1,2}$ D. Pandit, ${ }^{1}$ D. Mandal, ${ }^{1}$ and S. R. Banerjee ${ }^{1,2}$ \\ ${ }^{1}$ Variable Energy Cyclotron Centre, 1/AF Bidhan Nagar, Kolkata 700064, India \\ ${ }^{2}$ Homi Bhabha National Institute, Training School Complex, Anushaktinagar, Mumbai 400094, India \\ ${ }^{3}$ Department of Physics, Prabhat Kumar College, Contai 721404, India \\ ${ }^{4}$ Saha Institute of Nuclear Physics, $1 /$ AF Bidhan Nagar, Kolkata 700064, India \\ ${ }^{5}$ Department of Physics, Presidency University, Kolkata 700073, India \\ ${ }^{6}$ Department of Nuclear Physics, Research School of Physics and Engineering, The Australian National University, \\ Canberra, ACT 2601, Australia
}

(Received 26 March 2019; published 13 May 2019)

\begin{abstract}
The excited states of ${ }^{197} \mathrm{Tl}$ have been studied via ${ }^{197} \mathrm{Au}\left({ }^{4} \mathrm{He}, 4 \mathrm{n}\right){ }^{197} \mathrm{Tl}$ reaction at a beam energy of $50 \mathrm{MeV}$ from the K-130 cyclotron at the Variable Energy Cyclotron Centre (VECC). The $\gamma$ rays were detected using the VECC array for Nuclear Spectroscopy (VENUS) with six Compton-suppressed clover HPGe detectors. An improved level scheme of ${ }^{197} \mathrm{Tl}$ has been proposed from this work, which has been extended to $5.1 \mathrm{MeV}$ of excitation energy and 39/2 $\hbar$ of spin from the placement of 28 new $\gamma$-ray transitions. Band crossings in the known one- and three-quasiparticle (qp) bands have been identified for the first time in this work. Two new bands, based on 3-qp and 5-qp configurations, have been observed for the first time in this nucleus; both of which are identified as the magnetic rotational (MR) in nature. The excitation energies of these bands are similar to that of the doubly degenerate bands observed for the similar 3-qp and 5-qp configurations in ${ }^{195} \mathrm{Tl}$. These indicate a transition from an aplanar geometry of the neutron, proton, and the core angular momentum vectors to a planar one for neutron number $N \geqslant 116$ in $\mathrm{Tl}$ isotopes. The total Routhian surface calculations suggest a change in shape from oblate for the 1-qp configuration to a near-spherical one for the 3-and 5-qp configurations. This is consistent with the observed MR nature of the bands with multi-qp configuration. The MR bands are well reproduced by a model calculations in the frame work of the shears mechanism with the principal axis cranking.
\end{abstract}

DOI: 10.1103/PhysRevC.99.054312

\section{INTRODUCTION}

The proton Fermi surface of the Tl nuclei lies just below the $Z=82$ spherical shell closure. Odd- $A$ Tl nuclei have one proton hole in the $3 s_{1 / 2}$ orbital and accordingly, the ground-state spin-parity is $1 / 2^{+}[1-3]$. However, the high- $j$ proton orbitals such as $\pi h_{9 / 2}$ and $\pi i_{13 / 2}$, situated above the $Z=82$ shell closure, come down in energy with deformation parameter $\beta_{2}$ such that they lie close to the proton Fermi level and become accessible for the excitation of proton particles in Tl nuclei with small deformation. These orbitals are the so called intruder orbitals, which play important roles to break the spherical symmetry and induce deformations (both prolate and oblate) in a nucleus. Consequently, rotational bands based on the $\pi h_{9 / 2}$ and the $\pi i_{13 / 2}$ orbitals have been observed in the neutron deficient odd- $A$ Tl nuclei [4-17] from neutron number $N=101$ up to the neutron number as close as $N=120$ to the $N=126$ spherical shell closure.

On the other hand, in case of the neutron deficient $\mathrm{Tl}$ isotopes in $A \approx 190$ region, the neutron-hole excitations are possible in the high- $j$, shape-driving $i_{13 / 2}$ orbital around the

*gopal@vecc.gov.in neutron number $N=114$. Due to this, the moderate to highspin structures in $\mathrm{Tl}$ isotopes in $A \approx 195$ region are expected to be dominated by the particle-hole excitations, which give rise to different interesting phenomena related with the coupling of the particle and the hole angular momentum vectors with the core. Indeed, magnetic rotational (MR) bands and chiral doublet bands have been reported in several odd- $A$ and odd-odd Tl nuclei up to $N=117$ in this region [18-22]. However, no MR or chiral bands have been reported in ${ }^{199,200} \mathrm{Tl}$ $[14,15,23]$, which might indicate that the neutron number below $N=118$ puts a boundary in Tl nuclei for the observation of such interesting band structures emerges from the particlehole excitations in high- $j$ orbitals. This boundary may correspond to the energetically unfavoured excitations of neutron holes to the $v i_{13 / 2}$ orbital at or above this neutron number.

In this context, ${ }^{197} \mathrm{Tl}$ is an interesting nucleus to study. Existence of the nearly degenerate bands, manifested due to the chiral symmetry breaking in a triaxial nucleus with proton(s) and neutron(s) in the high- $j$ particle and hole angular momentum states, were predicted long ago in the nuclei in both the $A \approx 130$ and $A \approx 195$ regions [24,25]. Several such band structures have been experimentally identified in $A \approx 130$ region but the first experimental evidence in $A \approx 195$ region with lifetime measurements has been reported only 
recently in ${ }^{194} \mathrm{Tl}$ [19]. Degenerate bands, interpreted as due to the chiral symmetry breaking, have also been reported in ${ }^{198} \mathrm{Tl}$ [22] and in ${ }^{195} \mathrm{Tl}$ [21]. In case of ${ }^{195} \mathrm{Tl}$, not only that the doubly degenerate bands have been observed in an odd- $A$ nucleus in this region, but also, for the first time in this region, more than one chiral bands have been reported. The observation of multiple chiral doublet $(\mathrm{M} \chi \mathrm{D})$ bands, first predicted by Meng et al. [26], signifies the evidence of triaxial shape coexistence in ${ }^{195} \mathrm{Tl}$. Still the observed chiral doublet bands in this region are very few in number compared to that in the $A \approx 130$ region. A logical extension would, therefore, be to look for such doubly degenerate bands in other nuclei in $A \approx 195$ region. In ${ }^{197} \mathrm{Tl}$, the neutron Fermi level would lie around the $v i_{13 / 2}$ orbital and in between that of ${ }^{195} \mathrm{Tl}$ and ${ }^{198} \mathrm{Tl}$, for both of which doubly degenerate bands have been reported.

The high spin states in ${ }^{197} \mathrm{Tl}$ nucleus are known mostly from the ${ }^{4} \mathrm{He}$ and ${ }^{3} \mathrm{He}$ induced fusion evaporation reactions $[12,13,27]$. In these studies, two rotational bands, based on a one-quasiparticle (1-qp) and a 3-qp configuration have been reported. However, no band crossing phenomenon has been observed in any of these bands. So, the effect of the alignment of a pair of neutrons in these rotational-like bands could not be studied. It may be noted that the doubly degenerate bands in ${ }^{195} \mathrm{Tl}$ were observed after neutron alignments in the $i_{13 / 2}$ orbital. Apart from the $i_{13 / 2}$, the negative parity $f_{5 / 2}, p_{3 / 2}$, and $p_{1 / 2}$ orbitals are also available near the Fermi level for the nuclei with neutron number $N>114$. Therefore, it is important to study the higher spin states in ${ }^{197} \mathrm{Tl}$ beyond the neutron alignments in order to understand the type of band structures generated due to the neuron alignments in the positive and in the negative parity orbitals.

\section{EXPERIMENTAL DETAILS}

The excited states in ${ }^{197} \mathrm{Tl}$ were populated by the fusion evaporation reaction ${ }^{197} \mathrm{Au}\left({ }^{4} \mathrm{He}, 4 \mathrm{n}\right){ }^{197} \mathrm{Tl}$ at $50 \mathrm{MeV}$ of beam energy delivered from the K-130 cyclotron at the Variable Energy Cyclotron Centre, Kolkata (VECC). The self-supporting, $5 \mathrm{mg} / \mathrm{cm}^{2}$ thick ${ }^{197} \mathrm{Au}$ target was at an angle of about $55^{\circ}$ with respect to the beam direction. The in-beam $\gamma$ rays were detected using the VECC array for Nuclear Spectroscopy (VENUS) [15,28]. It consisted of six Compton-suppressed clover HPGe detectors in the horizontal plane at the time of the experiment. The detectors were arranged in four angles with respect to the beam direction with two each at $150^{\circ}$ and $90^{\circ}$; the other two were at $45^{\circ}$ and $55^{\circ}$ angles. The energy and the efficiency calibrations of the clover detectors were done using radioactive ${ }^{133} \mathrm{Ba}$ and ${ }^{152} \mathrm{Eu}$ sources. The data were recorded in the list mode format using VME-based data acquisition system with the requirement of $\gamma-\gamma$ coincidence master trigger. The time difference between the master gate and the RF signal of the cyclotron was also recorded in a time-to-amplitude converter (TAC) module. Some of the data files were recorded in singles mode for the intensity and the angular distribution measurement of the $\gamma$ rays.

\section{DATA ANALYSIS}

The Linux Advanced MulitParameter System (LAMPS) analysis package was used for sorting the raw data files

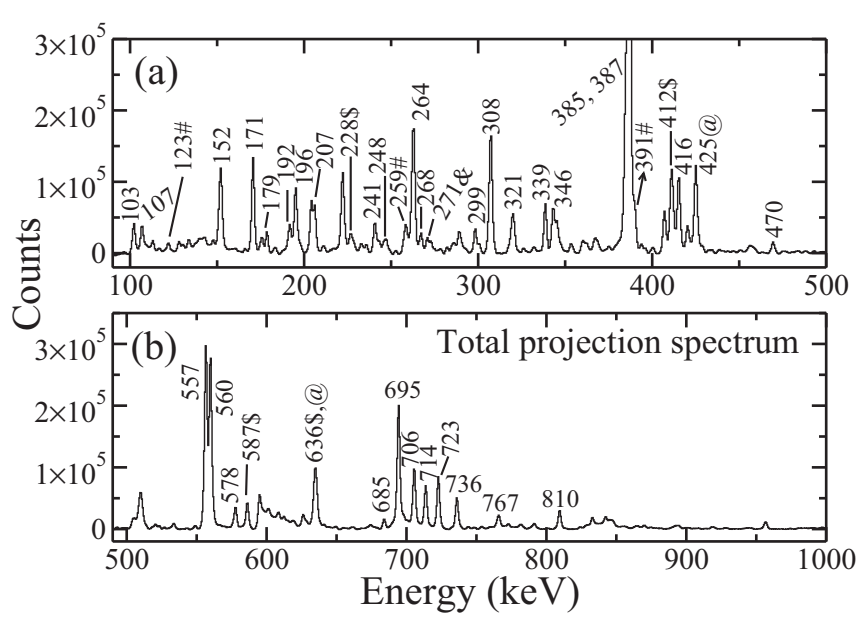

FIG. 1. The total projectrum spectrum from the $\gamma-\gamma$ matrix for the (a) lower and the (b) higher energy parts. The known $\gamma$-ray peaks from different nuclei are shown. The peaks with no symbol are the known peaks in ${ }^{197} \mathrm{Tl}$ while the symbols correspond to \#: ${ }^{198} \mathrm{Tl} ; \&$ : ${ }^{196} \mathrm{Tl}$; \$: ${ }^{198} \mathrm{Hg}$; and @: ${ }^{196} \mathrm{Hg}$.

[29]. Several $\gamma-\gamma$ matrices were generated from the data for the analysis. The $\gamma-\gamma$ matrices were analyzed by using the RADWARE [30] analysis package to construct the level scheme of ${ }^{197} \mathrm{Tl}$. A total $\gamma-\gamma$ matrix was generated with a prompt coincidence time window of $90 \mathrm{~ns}$ between the addback events of the clovers, which contained $1.7 \times 10^{8} \gamma-\gamma$ coincidence events. A total projection spectrum from this total $\gamma-\gamma$ matrix has been shown in Fig. 1. It shows the known peaks from ${ }^{197} \mathrm{Tl}$ as well as the stronger peaks in the other nuclei produced in the present experiment. It can be seen that the production of the other channels are much lower compared to ${ }^{197} \mathrm{Tl}$. It may be noted that a few low-lying transitions from the even-even ${ }^{196,198} \mathrm{Hg}$ nuclei, produced as $(p, x n)$ channel, are also observed in this spectrum.

The multipolarities of the $\gamma$ rays were assigned from the measurement of directional correlation from oriented states (DCO) ratio [31] and the angular distribution measurements. In DCO ratio analysis, an asymmetric matrix was made with data from the $150^{\circ}$ detectors $\left(\theta_{1}\right)$ on one axis and from the $90^{\circ}$ detectors $\left(\theta_{2}\right)$ on the other axis. The DCO ratio $\left(R_{\mathrm{DCO}}\right)$ for $\gamma_{1}$, gated by another $\gamma$-ray $\gamma_{2}$ of known multipolarity, was obtained from the ratio of the intensities of $\gamma_{1}$ at two different angles $\theta_{1}$ and $\theta_{2}$ as,

$$
R_{\mathrm{DCO}}=\frac{I_{\gamma_{1}}\left(\text { Measured at } \theta_{1} ; \text { gated by } \gamma_{2} \text { at } \theta_{2}\right)}{I_{\gamma_{1}}\left(\text { Measured at } \theta_{2} ; \text { gated by } \gamma_{2} \text { at } \theta_{1}\right)} .
$$

The $R_{\mathrm{DCO}}$ value is close to unity $(0.5)$ for stretched transitions with same (different) multipolarities of $\gamma_{1}$ and $\gamma_{2}$. The value of $R_{\mathrm{DCO}}$ depends on the initial spin alignment, denoted by the width of the substate population $\sigma / J$, of the residual nucleus emitting the $\gamma$ ray and on the mixing ratio $(\delta)$, in case of a mixed transition. The value of $\sigma / J$ is expected to be wider in the $\alpha$-induced reactions, as in the present work, compared to the heavy-ion induced reaction for which $\sigma / J \approx$ 0.3 is normally used. Therefore, the value of $\sigma / J$ has been estimated, in this work, by comparing the experimental and 


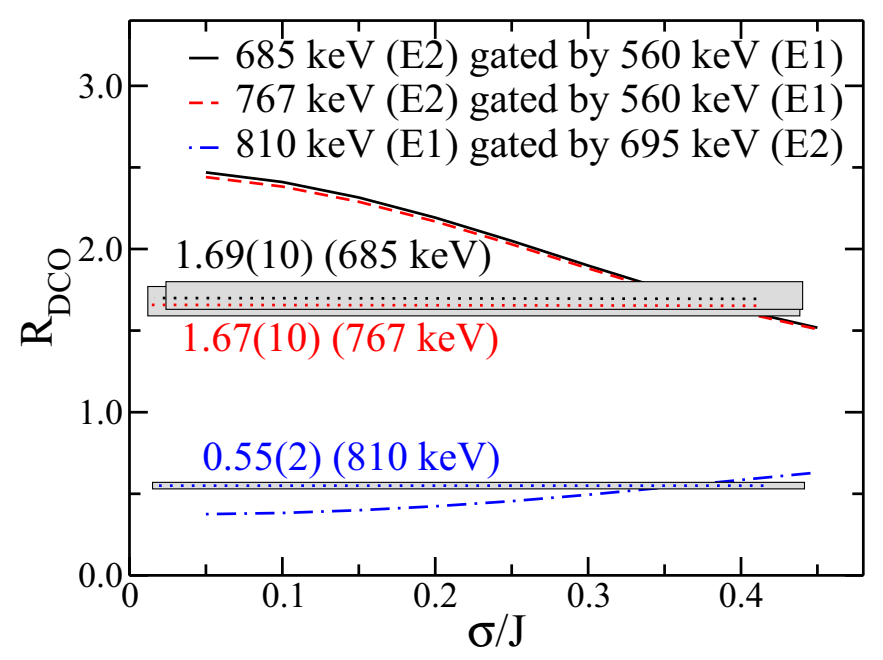

FIG. 2. Theoretical (solid, dashed, and dashed-dot lines) and measured $R_{\text {DCO }}$ values for different $(\sigma / J)$ for the three stretched $\gamma$ rays. The measured values are given and are shown as dotted lines with shaded regions encompass the uncertainties. The values of the $x$ axis corresponding to the crossing of the theoretical lines and the experimental ones are taken as the experimentally determined $\sigma / J$.

calculated values of $R_{\mathrm{DCO}}$ of some of the stretched transitions with known multipolarities. These are shown in Fig. 2. The $R_{\text {DCO }}$ values for these transitions have been calculated using the ADRAP code [32] for different values of $\sigma / J$. This gives similar values of $\sigma / J$ for the three transitions, namely, $\sigma / J=$ $0.38(4), 0.37(4), 0.37(2)$ corresponding to $685-\mathrm{keV}(E 2)$, $766-\mathrm{keV}(E 2)$, and $810-\mathrm{keV}(E 1)$ transitions, respectively (see Fig. 2). So, a weighted average value of $\sigma / J=0.37(3)$ has been adopted for this work.

The type $(E / M)$ of a transition has been obtained from the polarization measurement to determine the parity of a state. To measure the linear polarization, data from the clover detectors at $90^{\circ}$ have been used. The linear polarization $(P)$ is defined as [33] $P=\Delta_{\mathrm{PDCO}} / Q$, where, $Q$ is the polarization sensitivity and $\Delta_{\mathrm{PDCO}}$ is the measured polarization asymmetry, the value of which gives an idea about the type of a radiation $(E / M)$ and is given by,

$$
\Delta_{\mathrm{PDCO}}=\frac{a\left(E_{\gamma}\right) N_{\perp}-N_{\|}}{a\left(E_{\gamma}\right) N_{\perp}+N_{\|}},
$$

where $N_{\perp}$ and $N_{\|}$are the Compton-scattered events in the planes perpendicular and parallel to the reaction plane, respectively. To measure $N_{\perp}$ and $N_{\|}$, two asymmetric matrices of $N_{\perp}$ vs. all detectors and $N_{\|}$vs. all detectors were generated. The asymmetric response of the clover segments was corrected by the factor $a\left(E_{\gamma}\right)\left(=\frac{N_{\|}}{N_{\perp}}\right)$, which needs to be determined for an unpolarized radioactive source. In this experiment we have used the decay radiations of ${ }^{197} \mathrm{Tl}$ nucleus from the target foil to estimate the exact value of $a\left(E_{\gamma}\right)$ in order to avoid any uncertainty due to the position of external source. The values of $a\left(E_{\gamma}\right)$ are shown in Fig. 3 along with the fit using the equation, $a\left(E_{\gamma}\right)=a+b E_{\gamma}$. This gives the values of the coefficients as, $a=0.920(7)$ and $b=1.9(5) \times 10^{-5}$. Positive and negative values of the polarization asymmetry

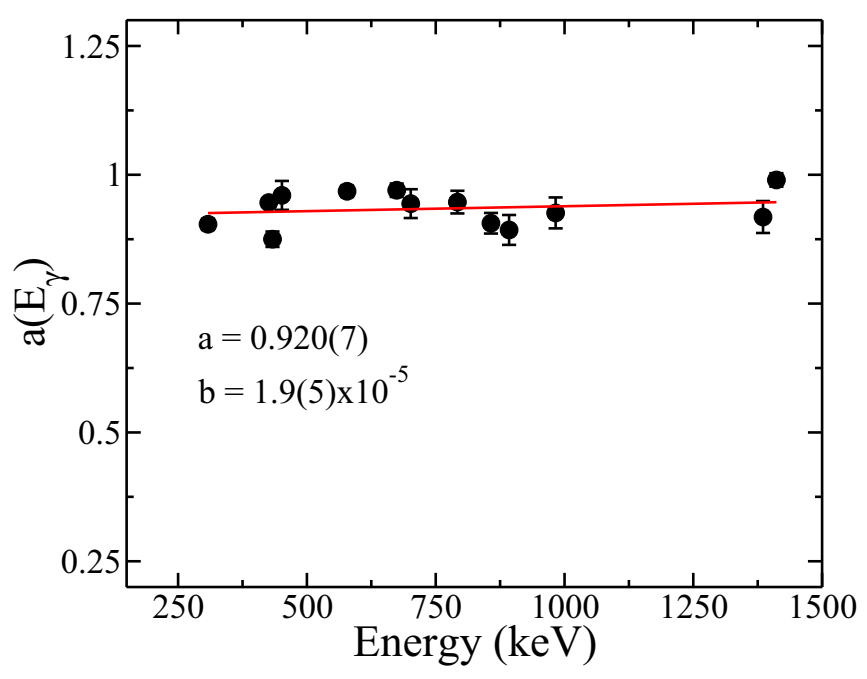

FIG. 3. Asymmetric correction factor $a\left(E_{\gamma}\right)$ for the detectors at $90^{\circ}$. solid line is the linear fit of the experimental data points. The values of coefficients $a$ and $b$ are also given.

$\Delta_{\text {PDCO }}$ indicate electric and magnetic types of the transitions, respectively. In the present setup, low-energy cut off for the $\Delta_{\text {PDCO }}$ measurement was about $180 \mathrm{KeV}$.

\section{EXPERIMENTAL RESULTS}

The experimental results of the $\gamma$-ray transition energies $\left(E_{\gamma}\right)$, level energies $\left(E_{i}\right)$, spins and parities of the initial $\left(J_{i}^{\pi}\right)$ levels, $R_{\mathrm{DCO}}$ and $\Delta_{\mathrm{PDCO}}$ values along with the adopted multipolarities of the $\gamma$-rays have been tabulated in Table I. The level scheme of ${ }^{197} \mathrm{Tl}$ as obtained in the present work is shown in Fig. 4. A total of 28 new $\gamma$ transitions have been identified for the first time and are placed in the level scheme. These are marked by asterisks $(*)$ in the level scheme. The level scheme has been extended up to an excitation energy of $5.14 \mathrm{MeV}$ and angular momentum of $19.5 \hbar$. The level scheme in Fig. 4 has been shown on top of the known $9 / 2^{-}$ isomer $\left[T_{1 / 2}=0.54(1) \mathrm{sec}\right]$ at $608 \mathrm{keV}$ of excitation energy [34].

The relative intensities of the $\gamma$ rays were determined from different single-gated spectra and all the intensities quoted in Table I are after proper normalization. The gated spectra were useful in estimating the intensities of the $\gamma$ rays of nearly degenerate energies which are present in different bands in ${ }^{197} \mathrm{Tl}$. These could be separated by choosing proper gating transitions.

\section{A. Level structure $A$}

The spin and parity of the level at $1953 \mathrm{keV}$ was assigned as $13 / 2^{+}$in the previous work [13]. This was based on the proposed $E 1$ nature of the $957-\mathrm{keV} \gamma$ ray. However, in the absence of any coincident stretched transition for gating, the assignment of dipole multipolarity of this transition, deduced from the $R_{\mathrm{DCO}}$ values gated by a mixed $(M 1+E 2)$ transition $(387 \mathrm{keV})$, was somewhat tentative; the electric $(E)$ nature of this transition was, however, confirmed from the $\Delta_{\text {PDCO }}$ measurement. Due to the lack of detectors, the angular distribution 
TABLE I. List of $\gamma$ rays belonging to ${ }^{197} \mathrm{Tl}$ with their energies $\left(E_{\gamma}\right)$ and intensities $\left(I_{\gamma}\right)$. The placement of these $\gamma$ rays in the level scheme are denoted by the energy $\left(E_{i}\right)$ and spin-parity $\left(J_{i}^{\pi}\right)$ of the decaying state. The measured values of $R_{\mathrm{DCO}}$ and $\Delta_{\mathrm{PDCO}}$ along with the adopted multipolarities are also given.

\begin{tabular}{|c|c|c|c|c|c|c|}
\hline$E_{\gamma}(\mathrm{keV})$ & $E_{i}(\mathrm{keV})^{\mathrm{i}}$ & $J_{i}^{\pi}$ & $I_{\gamma}^{a}$ & $R_{\mathrm{DCO}}(\mathrm{Err})$ & $\Delta_{\mathrm{PDCO}}(\mathrm{Err})$ & Multipolarity \\
\hline $102.5(2)$ & $3166.9(6)$ & $27 / 2^{-}$ & $1.22(5)$ & $0.65(6)^{\mathrm{a}}$ & & $M 1+E 2$ \\
\hline 107.1(2) & $3273.9(7)$ & $29 / 2^{-}$ & $1.05(5)$ & $0.46(6)^{\mathrm{a}}$ & & $M 1+E 2$ \\
\hline $113.4(3)$ & $3871.4(7)$ & $29 / 2^{+}$ & $0.63(3)$ & $0.61(5)^{\mathrm{f}}$ & & $M 1+E 2$ \\
\hline $152.3(2)$ & $2264.9(4)$ & $17 / 2^{+}$ & $5.02(9)$ & $1.04(2)^{\mathrm{e}}$ & & $M 1+E 2$ \\
\hline 161.1(4) & $2542.7(7)$ & $17 / 2^{+}$ & $0.16(1)$ & $1.05(13)^{\mathrm{d}}$ & & $M 1+E 2$ \\
\hline $170.9(1)$ & $2594.8(3)$ & $21 / 2^{-}$ & 7.04(11) & $0.53(2)^{a}$ & & $M 1+E 2$ \\
\hline $176.2(5)$ & $4881.8(11)$ & $37 / 2^{+}$ & $0.65(3)$ & $0.51(4)^{\mathrm{f}}$ & & $M 1+E 2$ \\
\hline 178.9(3) & $3141.6(5)$ & $23 / 2^{+}$ & $1.31(5)$ & $0.64(5)^{\mathrm{h}}$ & & $M 1+E 2$ \\
\hline 192.1(4) & $3758.1(5)$ & $27 / 2^{+}$ & $1.36(5)$ & $1.00(7)^{\mathrm{e}}$ & & $M 1+E 2$ \\
\hline 194.2(4) & $2818.5(10)$ & $19 / 2^{+}$ & $0.075(1)$ & $1.06(12)^{\mathrm{d}}$ & & $M 1+E 2$ \\
\hline $195.5(2)$ & $2460.5(4)$ & $19 / 2^{+}$ & $5.02(9)$ & $0.90(2)^{\mathrm{e}}$ & $-0.05(2)$ & $M 1+E 2$ \\
\hline 197.1(3) & $4563.9(8)$ & $35 / 2^{+}$ & $0.11(1)$ & & & $(M 1+E 2)$ \\
\hline 204.5(3) & $4075.9(8)$ & $31 / 2^{+}$ & $2.51(8)$ & $0.58(6)^{\mathrm{f}}$ & $-0.30(12)$ & $M 1(+E 2)$ \\
\hline $204.7(2)$ & $3310.9(7)$ & $27 / 2^{+}$ & $0.63(2)$ & $0.50(7)^{\mathrm{e}}$ & $-0.13(8)$ & $M 1(+E 2)$ \\
\hline 204.7(3) & $3614.1(7)$ & $27 / 2^{+}$ & $0.25(1)$ & $0.51(6)^{\mathrm{h}}$ & & $M 1+E 2$ \\
\hline $206.6(5)$ & $3064.4(6)$ & $25 / 2^{-}$ & $3.14(9)$ & $0.64(4)^{\mathrm{b}}$ & $-0.23(5)$ & $M 1(+E 2)$ \\
\hline $210.9(3)$ & 2753.6(8) & $19 / 2^{+}$ & $0.11(1)$ & $0.96(10)^{\mathrm{d}}$ & & $M 1+E 2$ \\
\hline 241.1(2) & 2353.9(4) & $17 / 2^{+}$ & $2.99(7)$ & $1.01(5)^{\mathrm{e}}$ & $-0.28(8)$ & $M 1(+E 2)$ \\
\hline $242.8(6)$ & $2624.4(9)$ & $17 / 2^{+}$ & $0.18(1)$ & $1.06(16)^{\mathrm{d}}$ & & $M 1+E 2$ \\
\hline $247.9(3)$ & $3106.2(6)$ & $25 / 2^{+}$ & $0.95(4)$ & $0.47(5)^{\mathrm{b}}$ & $0.16(9)$ & $E 1$ \\
\hline 258.3(6) & $5140.1(12)$ & $39 / 2^{+}$ & $0.30(2)$ & $0.51(6)^{\mathrm{f}}$ & & $M 1+E 2$ \\
\hline $260.1(4)$ & $2624.4(9)$ & $17 / 2^{+}$ & $0.05(2)$ & & & $(M 1+E 2)$ \\
\hline $262.2(3)$ & $4338.1(8)$ & $33 / 2^{+}$ & $2.42(8)$ & $0.53(5)^{\mathrm{f}}$ & $-0.22(7)$ & $M 1(+E 2)$ \\
\hline $263.6(4)$ & 2858.4(5) & $23 / 2^{-}$ & $8.60(20)$ & $0.44(2)^{\mathrm{a}}$ & $-0.14(5)$ & $M 1(+E 2)$ \\
\hline $262.9(3)$ & $3404.5(6)$ & $25 / 2^{+}$ & $0.61(2)$ & $0.43(6)^{\mathrm{h}}$ & $-0.22(8)$ & $M 1(+E 2)$ \\
\hline $263.1^{j}$ & $2528.0(2)$ & $19 / 2^{+}$ & $0.17(6)$ & & & $M 1+E 2$ \\
\hline $267.8(3)$ & $3409.4(6)$ & $25 / 2^{+}$ & $0.79(4)$ & $0.89(7)^{\mathrm{e}}$ & $-0.1(1)$ & $M 1+E 2$ \\
\hline 273.1(3) & $3584.1(7)$ & $29 / 2^{+}$ & $0.68(2)$ & $0.50(5)^{\mathrm{b}}$ & $-0.14(8)$ & $M 1(+E 2)$ \\
\hline $286.2(3)$ & $3560.2(7)$ & $31 / 2^{-}$ & $0.91(7)$ & $0.55(5)^{\mathrm{b}}$ & $-0.33(12)$ & $M 1(+E 2)$ \\
\hline $288.9(4)$ & 2962.7(4) & $21 / 2^{+}$ & $2.33(6)$ & $0.91(5)^{\mathrm{e}}$ & $-0.12(5)$ & $M 1(+E 2)$ \\
\hline $298.9(2)$ & $2016.9(3)$ & $17 / 2^{-}$ & $6.05(9)$ & $0.38(5)^{\mathrm{a}}$ & $-0.11(4)$ & $M 1+E 2$ \\
\hline $307.6(3)$ & $1302.6(2)$ & $13 / 2^{-}$ & $19.53(3)$ & $0.31(1)^{\mathrm{b}}$ & $-0.10(4)$ & $M 1+E 2$ \\
\hline $319.8(1)$ & 2673.7(4) & $19 / 2^{+}$ & $2.56(8)$ & $0.91(4)^{\mathrm{e}}$ & $-0.09(4)$ & $M 1(+E 2)$ \\
\hline $320.5(2)$ & 2038.8(3) & $17 / 2^{-}$ & $3.41(8)$ & $0.39(5)^{\mathrm{a}}$ & $-0.03(10)$ & $M 1+E 2$ \\
\hline $338.9(2)$ & 2799.4(4) & $21 / 2^{+}$ & $5.73(11)$ & $0.46(2)^{\mathrm{f}}$ & $-0.04(5)$ & $M 1+E 2$ \\
\hline $345.8(3)$ & $3145.2(4)$ & $23 / 2^{+}$ & $3.15(9)$ & $1.00(6)^{\mathrm{e}}$ & $-0.15(17)$ & $M 1+E 2$ \\
\hline $348.1(2)$ & $2460.5(4)$ & $19 / 2^{+}$ & $0.72(2)$ & $1.02(7)^{\mathrm{f}}$ & & $E 2$ \\
\hline $360.9(3)$ & $3323.5(5)$ & $23 / 2^{+}$ & $0.95(4)$ & $1.18(6)^{\mathrm{h}}$ & $-0.09(8)$ & $M 1+E 2$ \\
\hline $362.8(3)$ & $3946.8(8)$ & $31 / 2^{+}$ & $0.54(3)$ & $0.45(5)^{\mathrm{b}}$ & & $M 1+E 2$ \\
\hline $367.5(6)$ & $4705.6(9)$ & $35 / 2^{+}$ & $0.95(1)$ & $0.49(6)^{\mathrm{f}}$ & $-0.11(5)$ & $M 1(+E 2)$ \\
\hline $385.2(2)$ & 2423.9(3) & $19 / 2^{-}$ & $6.08(34)$ & $0.56(1)^{\mathrm{a}}$ & $-0.03(3)$ & $M 1+E 2$ \\
\hline $387.2(3)$ & 995.1(2) & $11 / 2^{-}$ & $100.0(1)$ & $0.33(4)^{\mathrm{b}}$ & $-0.05(1)$ & $M 1+E 2$ \\
\hline 394.4(2) & $2112.5(3)$ & $15 / 2^{+}$ & $0.90(5)$ & $0.93(14)^{\mathrm{f}}$ & & $E 1$ \\
\hline $407.0(2)$ & $2423.9(3)$ & $19 / 2^{-}$ & $5.70(11)$ & $0.26(2)^{a}$ & $-0.19(4)$ & $M 1(+E 2)$ \\
\hline $412.3(4)$ & $3972.4(7)$ & $33 / 2^{-}$ & $0.36(3)$ & $0.46(3)^{\mathrm{b}}$ & & $M 1+E 2$ \\
\hline $411.2(6)$ & $2363.8(7)$ & $15 / 2^{+}$ & $0.61(3)$ & $0.72(4)^{\mathrm{d}}$ & & $M 1+E 2$ \\
\hline $415.5(6)$ & 1718.1(2) & $15 / 2^{-}$ & $12.67(16)$ & $0.25(1)^{\mathrm{a}}$ & $-0.08(4)$ & $M 1+E 2$ \\
\hline $420.9(3)$ & $3566.1(5)$ & $25 / 2^{+}$ & $1.97(8)$ & $0.38(2)^{g}$ & & $M 1+E 2$ \\
\hline $420.7(2)$ & $4367.3(8)$ & $33 / 2^{+}$ & $0.34(1)$ & $0.58(5)^{\mathrm{b}}$ & & $M 1+E 2$ \\
\hline 429.1(3) & $2381.6(7)$ & $15 / 2^{+}$ & $0.66(3)$ & $0.76(3)^{\mathrm{d}}$ & $-0.05(5)$ & $M 1+E 2$ \\
\hline $434.7(4)$ & $3758.1(5)$ & $27 / 2^{+}$ & $0.48(3)$ & $1.41(20)^{\mathrm{e}}$ & & $E 2$ \\
\hline $469.6(5)$ & $3064.4(6)$ & $25 / 2^{-}$ & $1.51(5)$ & $0.98(8)^{a}$ & $0.07(7)$ & $E 2$ \\
\hline $478.0(2)$ & $3584.2(7)$ & $29 / 2^{+}$ & $0.05(1)$ & & & \\
\hline $534.5(2)$ & 2799.4(4) & $21 / 2^{+}$ & $1.72(4)$ & $0.87(11)^{\mathrm{f}}$ & $0.02(1)$ & $E 2$ \\
\hline $555.7(2)$ & $2594.8(3)$ & $21 / 2^{-}$ & $3.73(11)$ & $1.09(6)^{a}$ & $0.11(4)$ & $E 2$ \\
\hline
\end{tabular}


TABLE I. (Continued).

\begin{tabular}{|c|c|c|c|c|c|c|}
\hline$E_{\gamma}(\mathrm{keV})$ & $E_{i}(\mathrm{keV})^{\mathrm{i}}$ & $J_{i}^{\pi}$ & $I_{\gamma}^{a}$ & $R_{\mathrm{DCO}}(\mathrm{Err})$ & $\Delta_{\mathrm{PDCO}}(\mathrm{Err})$ & Multipolarity \\
\hline $556.9(8)$ & $1552.3(4)$ & $13 / 2^{-}$ & $47.31(3)$ & $0.23(1)^{\mathrm{g}}$ & $0.05(3)$ & $M 1+E 2$ \\
\hline $560.1(1)$ & $2112.5(3)$ & $15 / 2^{+}$ & $47.13(6)$ & $0.53(2)^{g}$ & $0.15(3)$ & $E 1$ \\
\hline $578.1(4)$ & 2594.8(3) & $21 / 2^{-}$ & $4.19(11)$ & $0.92(5)^{\mathrm{a}}$ & $0.09(4)$ & $E 2$ \\
\hline $608.8(2)$ & $2962.7(4)$ & $21 / 2^{+}$ & $1.25(7)$ & $1.73(28)^{\mathrm{e}}$ & $0.2(1)$ & $E 2$ \\
\hline $612.8(7)$ & $3758.1(5)$ & $27 / 2^{+}$ & $1.40(5)$ & $1.89(22)^{\mathrm{e}}$ & $0.1(1)$ & $E 2$ \\
\hline $635.9(6)$ & $3946.8(8)$ & $31 / 2^{+}$ & $0.09(1)$ & $0.86(14)^{\mathrm{c}}$ & & $E 2$ \\
\hline $684.7(4)$ & $3145.2(4)$ & $23 / 2^{+}$ & $1.36(7)$ & $1.69(10)^{\mathrm{e}}$ & $0.2(1)$ & $E 2$ \\
\hline $694.6(3)$ & $1302.6(2)$ & $13 / 2^{-}$ & $35.30(8)$ & $1.09(3)^{\mathrm{b}}$ & $0.12(4)$ & $E 2$ \\
\hline $698.4(4)$ & $3972.4(7)$ & $33 / 2^{-}$ & $2.08(20)$ & $1.0(2)^{\mathrm{a}}$ & $0.22(11)$ & $E 2$ \\
\hline $705.8(2)$ & $2423.9(3)$ & $19 / 2^{-}$ & $15.77(23)$ & $1.09(3)^{\mathrm{a}}$ & $0.12(3)$ & $E 2$ \\
\hline 714.4(4) & $2016.9(3)$ & $17 / 2^{-}$ & $12.54(22)$ & $1.04(2)^{\mathrm{a}}$ & $0.08(2)$ & $E 2$ \\
\hline $782.3(6)$ & $4367.3(8)$ & $33 / 2^{+}$ & $0.14(1)$ & $1.05(14)^{\mathrm{c}}$ & & $E 2$ \\
\hline $809.8(3)$ & $2112.5(3)$ & $15 / 2^{+}$ & $7.60(16)$ & $0.55(2)^{\mathrm{a}}$ & $0.09(3)$ & $E 1$ \\
\hline $871.2(5)$ & $1866.3(6)$ & $13 / 2^{-}$ & $0.63(4)$ & & $-0.27(18)$ & $M 1+E 2$ \\
\hline $957.4(6)$ & $1952.5(7)$ & $13 / 2^{+}$ & $8.58(10)$ & & $0.13(4)$ & $E 1$ \\
\hline
\end{tabular}

a From the 695-keV (E2) gate.

${ }^{\mathrm{b}}$ From the 737-keV (E2) gate.

${ }^{\mathrm{c}}$ From the 706-keV (E2) gate.

${ }^{\mathrm{d}}$ From the 957-keV $(E 1)$ gate.

${ }^{\mathrm{e}}$ From the $560-\mathrm{keV}(E 1)$ gate.

${ }^{\mathrm{f}}$ From the 767-keV (E2) gate.

${ }^{\mathrm{g}}$ From the 685-keV (E2) gate.

${ }^{\mathrm{h}}$ From the 609-keV (E2) gate.

${ }^{\mathrm{i}}$ Least-square fit using the GTOL code of ENSDF [35].

jAdopted from Ref. [12].

B

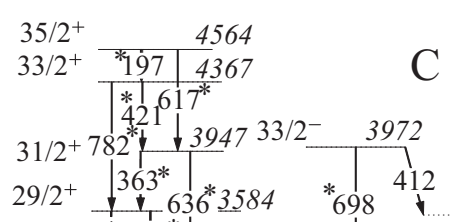

$\left.27 / 2^{+}+478\right)^{2} 3^{*}+331129 / 2^{-} \downarrow 3274 \quad 286$

$\mathrm{D}$

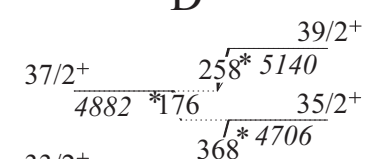

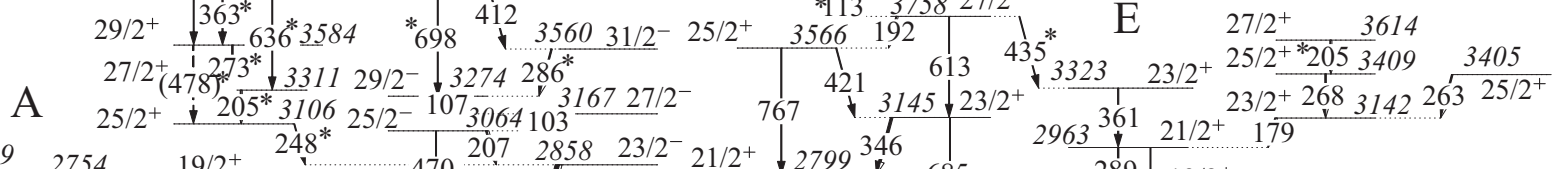

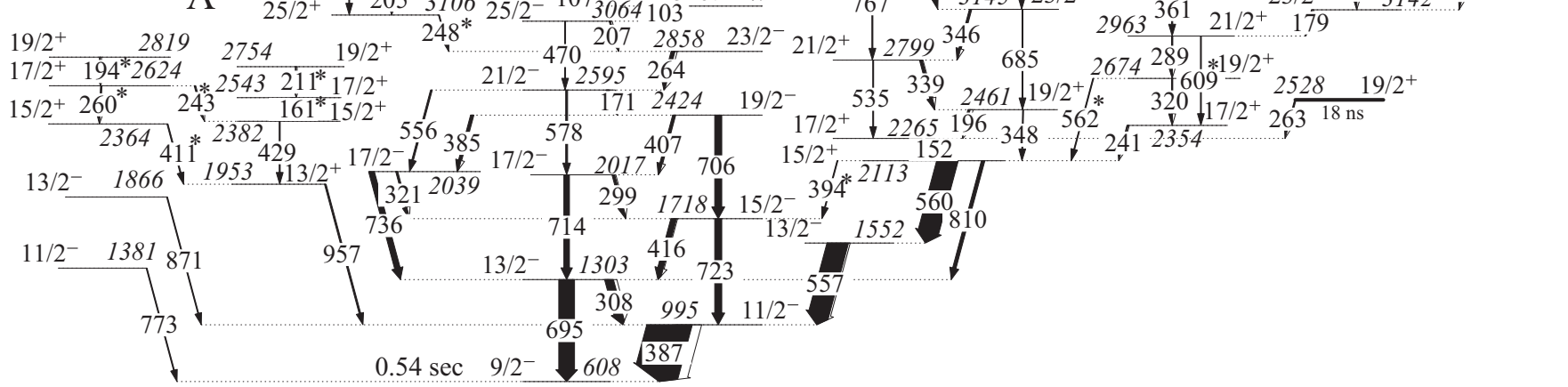

FIG. 4. Proposed level scheme of ${ }^{197} \mathrm{Tl}$ from the present work. Levels above the $0.54-\mathrm{sec}$ isomer at $608 \mathrm{keV}$ are shown. The new $\gamma$ transitions are marked by asterisks $(*)$. 


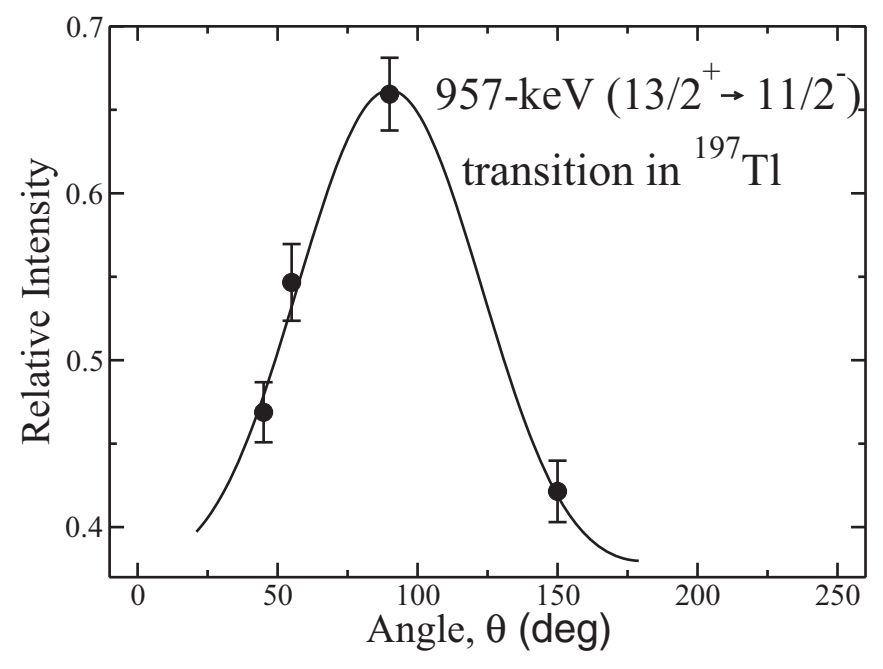

FIG. 5. Angular distribution of the $957-\mathrm{keV}$ transition in ${ }^{197} \mathrm{Tl}$ from the singles-data. The solid line is the fitted curve for a dipole transition.

measurements could not be performed in the previous work. The angular distribution of this $\gamma$ ray, from the data taken in the singles mode, has been measured in the present work and is shown in Fig. 5. The $\Delta_{\text {PDCO }}$ value, obtained in this work also agrees to that in Ref. [13]. Therefore, the $E 1$ nature of the $957-\mathrm{keV} \gamma$ ray and thereby, the $13 / 2^{+}$assignment of the $1953-\mathrm{keV}$ level in ${ }^{197} \mathrm{Tl}$ has been confirmed.

The $429-\mathrm{keV} \gamma$ ray, on top of the $13 / 2^{+}$state and decaying from the 2382-keV level, was known earlier [13] but with no spin-parity assignment for this level. In the present work, we have observed several new $\gamma$-ray transitions above $1953-\mathrm{keV}$ and $2382-\mathrm{keV}$ levels. These are grouped in to a bandlike structure $A$. The sum-gated spectrum with gates on 957- and 429-keV $\gamma$ rays, shown in Fig. 6, shows all the transitions belonging to this bandlike structure. The $R_{\mathrm{DCO}}$ values of these transitions, shown in Table I, are based on the $957-\mathrm{keV}$ E1 gate.

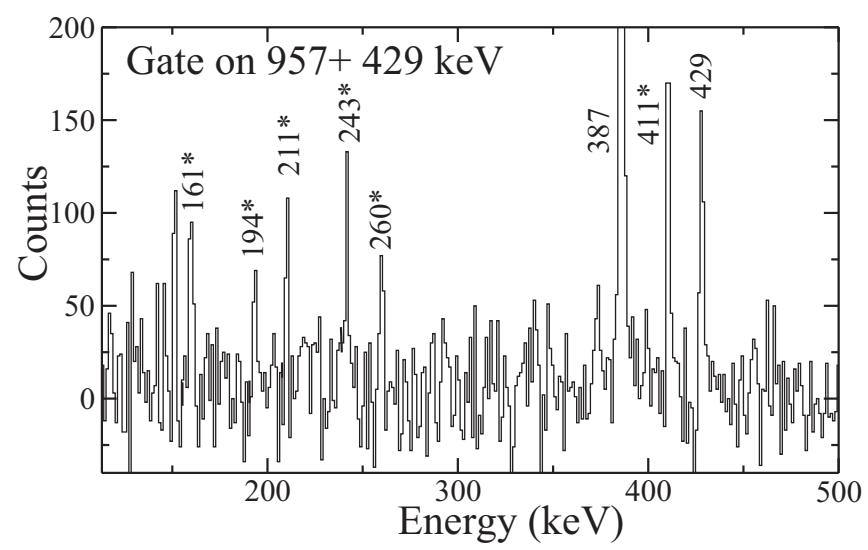

FIG. 6. Sum-gated spectrum with gates on 957-keV and 429-keV transitions corresponding to the bandlike structure $\mathrm{A}$ in ${ }^{197} \mathrm{Tl}$; new $\gamma$ rays are marked by asterisks $(*)$.

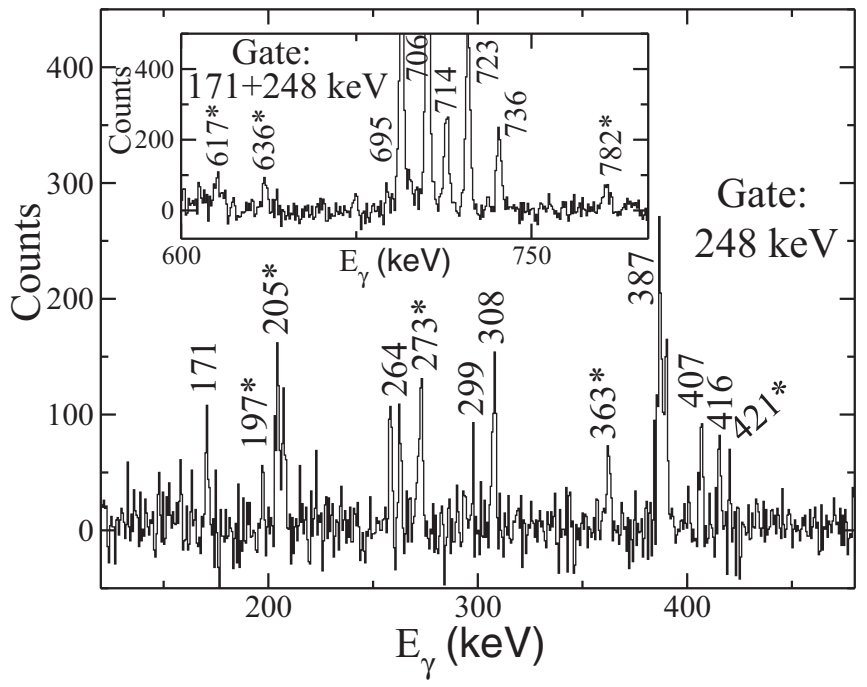

FIG. 7. A spectrum gated by $248 \mathrm{keV} \gamma$ ray showing the $\gamma$ rays in band $B$. The higher-energy $\gamma$ lines in band $B$ are shown in the sum gated $(171+248 \mathrm{keV})$ spectrum in the inset. The new $\gamma$ rays are marked by asterisks $(*)$.

\section{B. Band structures $B$ and $C$}

The band $B$ has been identified for the first time in this work while a few new transitions have been observed and placed at the top of the band $C$. The newly observed $\gamma$-rays in band $B$ are shown in the spectrum of Fig. 7 obtained by gating on the $248-\mathrm{keV}$ connecting transition. The weak $E 2$ crossover transitions of higher energies are shown in the inset of Fig. 7 in a sum gate of 171- and $248-\mathrm{keV}$ transitions. The $248-\mathrm{keV} \gamma$ ray has been observed in the sum-gated spectrum of Fig. 8 corresponding to the band $C$ and also in coincidence with other $\gamma$-rays of band $C$. The placement of the $248-\mathrm{keV}$ $\gamma$ ray has been assigned from the fact that the $470-\mathrm{keV} \gamma$ ray

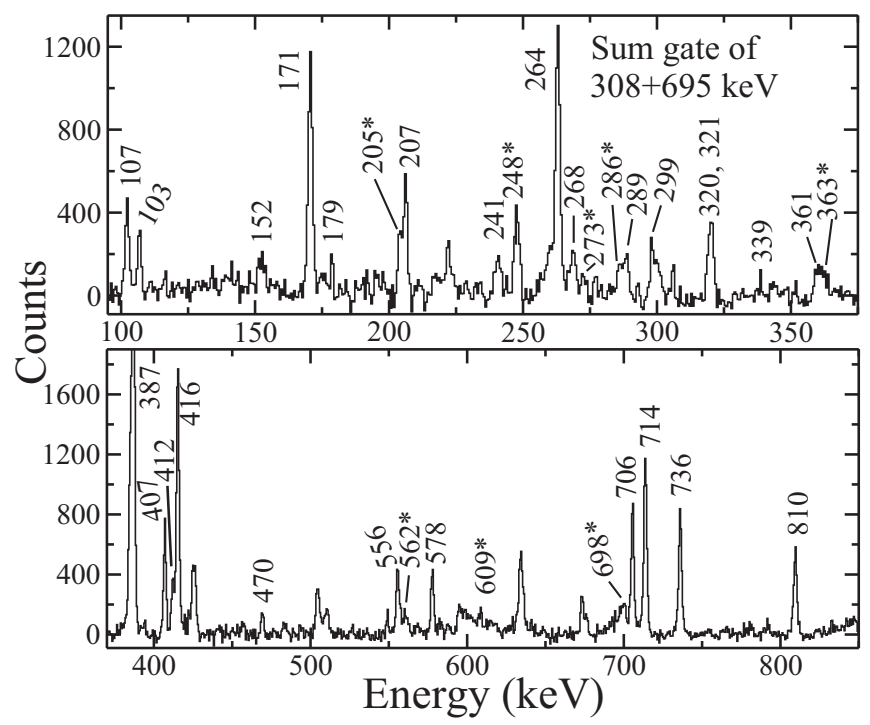

FIG. 8. Sum-gated spectrum with gates on $695-\mathrm{keV}$ and $308-\mathrm{keV}$ transitions corresponding to band $\mathrm{C}$ in ${ }^{197} \mathrm{Tl}$; new $\gamma$ rays are marked by asterisks $(*)$. 


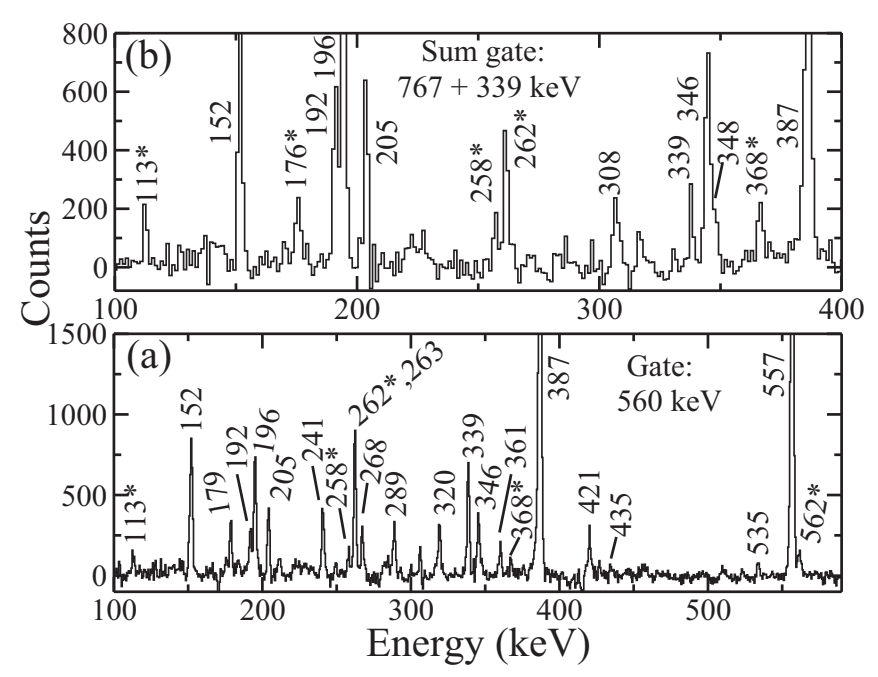

FIG. 9. (a) Coincidence spectrum gated by $560-\mathrm{keV} \gamma$ ray and (b) sum coincidence spectrum gated by $767-\mathrm{keV}$ and $339-\mathrm{keV} \gamma$ rays showing the transitions in the sequences $D$ and $E$. The new $\gamma$ rays are marked by asterisks $(*)$.

decaying from the 3064-keV, $25 / 2^{-}$level and the ones above it in band $C$ are not observed in the $248-\mathrm{keV}$ gated spectrum of Fig. 7. But the 171- and 264-keV $\gamma$-rays and the ones below it are observed in that spectrum. The $R_{\mathrm{DCO}}$ and the polarisation asymmetry values obtained for the $248-\mathrm{keV}$ transition (see Table I) clearly indicate that it is an $E 1$ transition. Hence, the spin-parity $\left(J^{\pi}\right)$ of the band head of the band $B$ at $3106 \mathrm{keV}$ has been assigned as $J^{\pi}=25 / 2^{+}$.

Most of the transitions in band $C$ were known from the previous works and are confirmed in this work. The $\gamma$ rays in this band are shown in Fig. 8. In one of the earlier works, a $412-\mathrm{keV} \gamma$ ray was placed on top of the $3274-\mathrm{keV}$ level by Lieder et al. [12], which has been given a slightly different placement in the present work, but consistent with the earlier placement. The $286-\mathrm{keV}$ and the crossover $698-\mathrm{keV}$ transitions have been identified and placed for the first time in this work. The $R_{\mathrm{DCO}}$ values, gated by the stretched $E 2$ transitions, indicate that the 286 and the $412 \mathrm{keV}$ transitions are predominantly dipole in nature, whereas the cross over $698 \mathrm{keV}$ is a quadrupole one.

\section{Band structures $D, E$, and $F$}

The spectrum in Fig. 9(a), gated by the $560-\mathrm{keV}$ transition, shows the $\gamma$ rays in band $D, E$, and $F$. The transitions in the lower part of the band $D$ were known earlier but in the present work, it has been extended up to $39 / 2^{+}$state through the observation of a series of new $\gamma$ rays above the $27 / 2^{+}$ state. The new $\gamma$ lines in this band are shown in Fig. 9(b) which is a sum coincidence spectrum with gates on $767-\mathrm{keV}$ and 339-keV transitions. There are a few low-intensity $\gamma$ lines in this spectrum which could not be assigned as they do not satisfy all the coincidence conditions. The measured $R_{\mathrm{DCO}}$ and the $\Delta_{\text {PDCO }}$ values of the 557-, 560-, and the $810-\mathrm{keV}$ transitions are consistent with the positive parity assignment

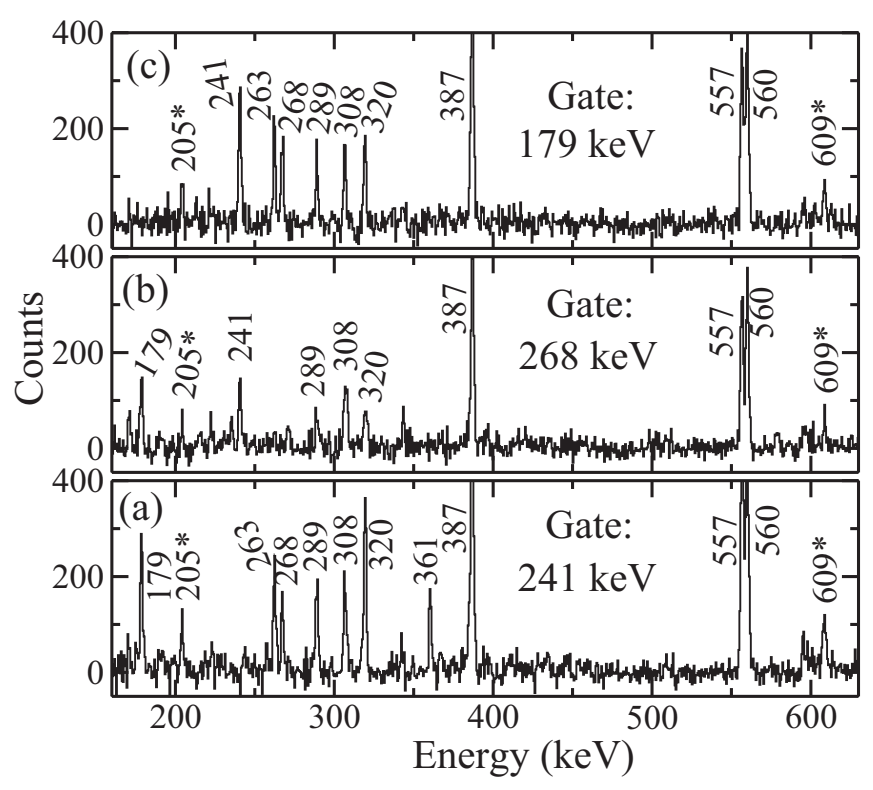

FIG. 10. Coincidence spectra gated by (a) $241-\mathrm{keV}$, (b) $268-\mathrm{keV}$, and (c) $179-\mathrm{keV}$ transitions showing the $\gamma$ lines placed in sequence $E$ and $F$ in the level scheme. New $\gamma$ rays are marked by asterisks (*).

for this band. It is interesting to note that no cross-over $E 2$ transition has been observed above $27 / 2^{+}$in this band.

In the earlier work [13] a new band (B3) was proposed above $2376-\mathrm{keV}$ of excitation energy which consisted of six transitions of energy 179, 241, 267, 289, 320, and $361 \mathrm{keV}$. This band was connected to the known states in ${ }^{197} \mathrm{Tl}$ by a $262-\mathrm{keV}$ transition. However, due to the lack of statistics, coincidence relation among these transitions could not be checked and, therefore, the placement of the $\gamma$ rays were somewhat tentative. In the present work, coincidence relations among these transitions could be thoroughly established due to higher statistics and it has been found that these $\gamma$ rays and a few new ones form two new sequences, denoted as $E$ and $F$ in Fig. 4. These sequences are built above a 2354-keV level which is connected to the known lower-lying levels by a $241-\mathrm{keV}$ transition. Figure 10 shows the coincidence relation for the transitions in sequences $E$ and $F$. The spectrum in Fig. 10(a) is gated by $241-\mathrm{keV}$ transition which shows all the $\gamma$ rays in sequences $E$ and $F$. Moreover, its intensity is the largest among the transitions in the sequences $E$ and $F$ in the spectrum of Fig. 9(a). Therefore, the $241-\mathrm{keV}$ transition is placed at the bottom of the two sequences $E$ and $F$.

The spectrum gated by 268-keV transition in Fig. 10(b) does not show any $\gamma$-ray peak at $263 \mathrm{keV}$, whereas, it is observed in both the spectra gated by $241-\mathrm{keV}$ and $179-\mathrm{keV}$ in Fig. 10(a) and in Fig. 10(c), respectively. Therefore, there must be a $263-\mathrm{keV}$ transition, which is parallel to $268 \mathrm{keV}$ but in coincidence with $241 \mathrm{keV}$ and $179 \mathrm{keV}$. So, it is placed above the $3142 \mathrm{keV}$ level and parallel to the sequence $F$. The intensity of the $263-\mathrm{keV}$ transition has been found to be much more (compared to the $268-\mathrm{keV}$ transition, for example) in Fig. 9(a) than in Fig. 10(a) and 10(c). This supports the multiple placement of the $263-\mathrm{keV}$ transition. The $361-\mathrm{keV}$ transition is observed in $241-\mathrm{keV}$ gate but it is not present in 


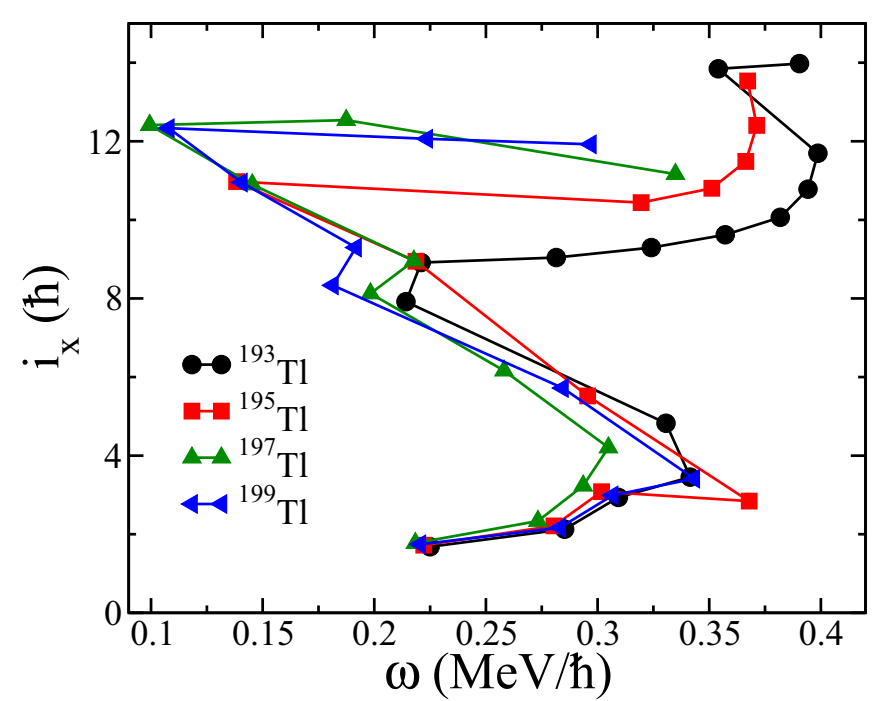

FIG. 11. Aligned angular momentum $i_{x}$ as a function of rotational frequency $(\omega)$ for the $\pi \mathrm{h}_{9 / 2}$ band in ${ }^{193,195,197,199} \mathrm{Tl}$. The Harris reference parameters are chosen to be $j_{0}=8 \hbar^{2} \mathrm{MeV}^{-1}$ and $j_{1}=$ $40 \hbar^{4} \mathrm{MeV}^{-3}$.

$268-\mathrm{keV}$ and $179-\mathrm{keV}$ gates. Therefore, the 179-keV, 268-keV and the newly observed $205-\mathrm{keV}$ transitions are placed to form the sequence $F$, parallel to the $361-\mathrm{keV}$ transition. A new $609-\mathrm{keV}$ crossover transition has been observed in sequence $E$ but no other such crossover transition is observed in these two sequences.

\section{DISCUSSION}

The ground state of all the $\mathrm{Tl}$ isotopes are $1 / 2^{+}$, corresponding to the odd-proton in the $3 s_{1 / 2}$ orbital and the first excited state is $3 / 2^{+}$, corresponding to the $\pi d_{3 / 2}$ orbital lying close to the proton Fermi level. The $9 / 2^{-}$state, corresponding to the intruder $\pi h_{9 / 2}$ orbital, is an isomeric state in the $\mathrm{Tl}$ isotopes. This configuration, coupled with the oblate deformed core of even-even $\mathrm{Hg}$, produces deformed rotational bands in odd- $A, \mathrm{Tl}$ isotopes. The rotational band based on the above configuration has been reported up to ${ }^{201} \mathrm{Tl}$ [17], which indicates that the shape driving effect of the $\pi h_{9 / 2}$ orbital still competes with the spherical shell closures even up to the neutron number $N=120$.

The band $C$ in Fig. 4 is the oblate deformed $\pi h_{9 / 2}$ band observed in all the odd- $A \mathrm{Tl}$ isotopes in $A=190$ region. The band crossing, due to the alignment of a pair of $i_{13 / 2}$ neutrons, has been observed by extending this band to higher spins in this work. So, the band crossing phenomenon can now be compared in all the neighboring odd- $A \mathrm{Tl}$ isotopes. Figure 11 shows the plot of the aligned angular momentum $\left(i_{x}\right)$ as a function of rotational frequency $(\omega)$ for the $\pi h_{9 / 2}$ band in ${ }^{197} \mathrm{Tl}$ and other neighboring $\mathrm{Tl}$ isotopes. The configuration of this band after the band crossing is $\pi h_{9 / 2} \otimes \nu i_{13 / 2}^{2}$.

The similarities in the band crossing frequency and alignment gain in all these isotopes indicate a common mechanism of $\nu i_{13 / 2}$ alignment. However, there are subtle differences both in the crossing frequencies and in the alignment gains in these

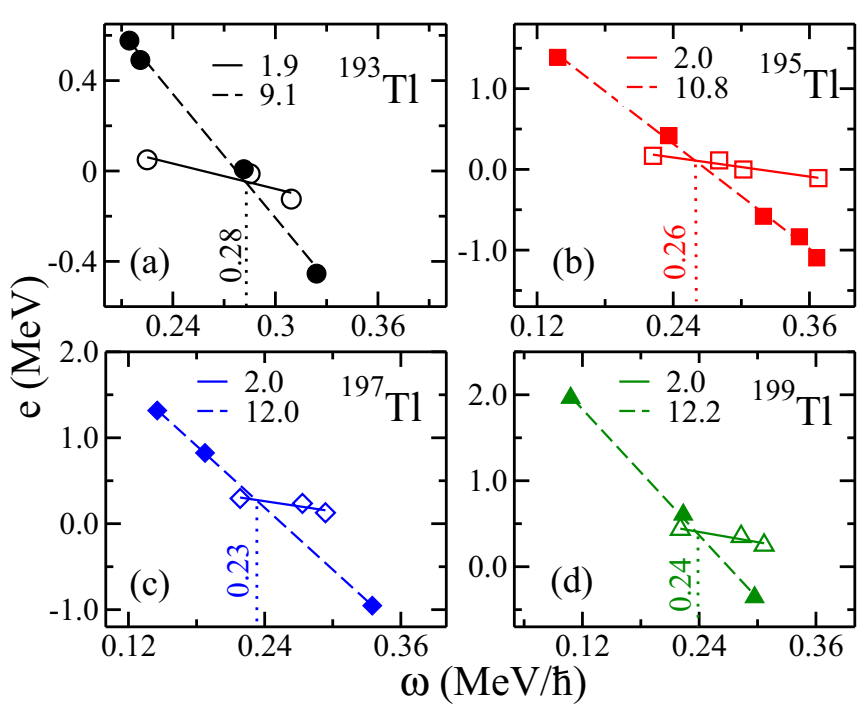

FIG. 12. Experimental Routhians (e) as a function of rotational frequency $(\omega)$ for the $\pi \mathrm{h}_{9 / 2}$ band in (a) ${ }^{193} \mathrm{Tl}$, (b) ${ }^{195} \mathrm{Tl}$, (c) ${ }^{197} \mathrm{Tl}$, and (d) ${ }^{199} \mathrm{Tl}$. The open and filled symbols correspond to the experimental data before and after the band crossing. The solid (dashed) lines are the linear fit to the data points before (after) the band crossing. The dotted lines denote the crossing point with the associated number is the crossing frequency, $\omega_{c}$. The values of the slopes of the two linear fits are also given for each isotope.

isotopes. In order to get a better quantitative values of the crossing frequency and the alignment gain, the experimental energy Routhians (e) are plotted as a function of rotational frequency $(\omega)$ in Fig. 12. In this plot the crossing frequencies are taken as the point of crossing of the two experimental Routhians corresponding to before and after the band crossings. These two regions were identified from Fig. 11. The slope of the Routhians gives the aligned angular momentum $\left(i_{x}\right)$, which is obtained by a linear fit of the data points in Fig. 12. It can be seen that the initial alignment of the $h_{9 / 2}$ band in all the $\mathrm{Tl}$ isotopes remain constant at a value of $i_{x} \approx 2 \hbar$. However, after the band crossing, $i_{x}$ increases from $\approx 9 \hbar$ in $N=112$ isotope ${ }^{193} \mathrm{Tl}$ to $\approx 12 \hbar$ in $N=116$ isotope ${ }^{197} \mathrm{Tl}$, which remains constant thereafter for $N=118$ isotope ${ }^{199} \mathrm{Tl}$. The crossing frequency, $\omega_{c}$, has been observed to decrease with the increase in neutron number until ${ }^{197} \mathrm{Tl}$ and remains almost same in ${ }^{199} \mathrm{Tl}$. This can be understood from the fact that as the neutron number increases, the neutron Fermi level moves from higher- $\Omega$ to lower- $\Omega$ orbitals in the Nilsson diagram for oblate deformation and, as a result, the required Coriolis force $\left(\omega_{c} i_{x}\right)$ can be achieved to align a pair of neutrons at a lower $\omega_{c}$. It may also be noted that $12 \hbar$ is the maximum angular momentum that can be obtained from the alignment of a pair of neutrons in $i_{13 / 2}$ orbital, which seems to be happening for $\mathrm{Tl}$ isotopes for neutron number $N \geqslant 116$, below which the $i_{13 / 2}$ neutrons are only partially aligned.

The states in ${ }^{197} \mathrm{Tl}$, which are denoted under the sequence $A$ in Fig. 4 are mostly noncollective and seem to have the single-particle origin. These are not extended much and do not seem to follow any collective pattern. These states decay to the $13 / 2^{+}$state at $1953 \mathrm{keV}$, which was identified as the 
$\pi i_{13 / 2}$ character [14]. The states above this may, therefore, be originated from the coupling of the $\pi i_{13 / 2}$ proton with the noncollective $2^{+}, 3^{+}$, and $4^{+}$states known in the neighboring even-even ${ }^{196} \mathrm{Hg}$ [36], which have most likely configuration of neutrons in the negative parity $f_{5 / 2}$ and $p_{3 / 2}$ orbitals. It is worthwhile to mention that it was possible to excite these noncollective states in ${ }^{197} \mathrm{Tl}$ as the light-ion $(\alpha)$ induced reaction has been used in the present experiment.

The band $B$ in Fig. 4 is a new band in ${ }^{197} \mathrm{Tl}$, which has been identified for the first time in this work. The excitation energy of this positive parity band indicates that it is based on a 3-qp configuration. This band decays to the negative parity yrast band, which has the configuration of $\pi h_{9 / 2} \otimes v i_{13 / 2}^{2}$. Therefore, considering the positive parity, the band $B$ has the possible configuration of $\pi i_{13 / 2} \otimes v i_{13 / 2}^{2}$.

From the measured intensities of the $\Delta I=1$ and $\Delta I=2$ transitions, the ratio of the $M 1$ and $E 2$ transition strengths, $B(M 1) / B(E 2)$, and thereby, the $g$ factors $\left(g_{k}\right)$ have been estimated for the $29 / 2^{+}, 31 / 2^{+}$, and the $33 / 2^{+}$states in band $B$ using the well-known relations given in Ref. [37]. This yields $g_{k}=0.57(4)-0.60(4)$ for the three states. In these estimations, the quadrupole moment $Q_{\circ}=3.0 \mathrm{eb}$, corresponding to a deformation of $\beta_{2} \approx 0.1$, was used. These experimental values of $g_{k}$ have excellent agreement with the calculated value of $g_{k}=0.58$ corresponding to the $\pi i_{13 / 2} \otimes \nu i_{13 / 2}^{2}$ configuration [37]. Hence, it provides a strong support to the assigned configuration of the band $B$.

In ${ }^{195} \mathrm{Tl}$, a negative parity partner band has been observed to the 3-qp ground band [21], but no such partner band has been observed in the present work. However, a band structure has been observed in ${ }^{195} \mathrm{Tl}$ at an excitation energy of $3138 \mathrm{keV}$ [38], which is similar to the band $B$ in ${ }^{197} \mathrm{Tl}$.

The excitation energy of the band $D$ is similar to the $5^{-}$band of the even-even core ${ }^{196} \mathrm{Hg}$ [39]. The $5^{-}$band in ${ }^{196} \mathrm{Hg}$ has the two-neutron hole configuration $v i_{13 / 2}^{-1} \otimes(p f)^{-1}$, where $(p f)$ denotes the negative parity orbitals, $3 p_{3 / 2}$ and $2 f_{5 / 2}$, situated around the Fermi level for neutron number $N=$ 116 [40]. A rotational band structure with both even and odd spin members has been observed to built on top of the $5^{-}$band head at $1757 \mathrm{keV}$ excitation energy in ${ }^{196} \mathrm{Hg}$. Considering the excitation energy of the $\pi h_{9 / 2}$ isomeric state $(608 \mathrm{keV})$ in ${ }^{197} \mathrm{Tl}$, the observed excitation energy of $2113 \mathrm{keV}$ for the band head $\left(15 / 2^{+}\right)$of band $D$ is quite consistent with the configuration of $\pi h_{9 / 2} \otimes v 5^{-}$. Rotational band structure has also been observed on top of this 3-qp band head in ${ }^{197} \mathrm{Tl}$, similar to that observed for ${ }^{199} \mathrm{Tl}[14]$.

The aligned angular momentum $\left(i_{x}\right)$ for this band has been shown in Fig. 13 as a function of rotational frequency $(\hbar \omega)$ along with that of the other neighboring nuclei. It can be seen that the initial gain in alignments of the odd- $A{ }^{197,199} \mathrm{Tl}$ nuclei are very similar to that of the $5^{-}$band of the even-even core ${ }^{194} \mathrm{Hg}$ [41] and ${ }^{196} \mathrm{Hg}$ [39]. This clearly indicates that there is very little contribution from the odd proton, which is consistent with the involvement of the $\Omega=9 / 2$ component of the $\pi h_{9 / 2}$ orbital for the oblate deformation.

This 3-qp band has been extended in the present work and it has been observed that this band is crossed by a 5-qp band above $3566 \mathrm{keV}$ (see Fig. 4). In the even-even core of $\mathrm{Hg}$, this crossing has been interpreted as due to the

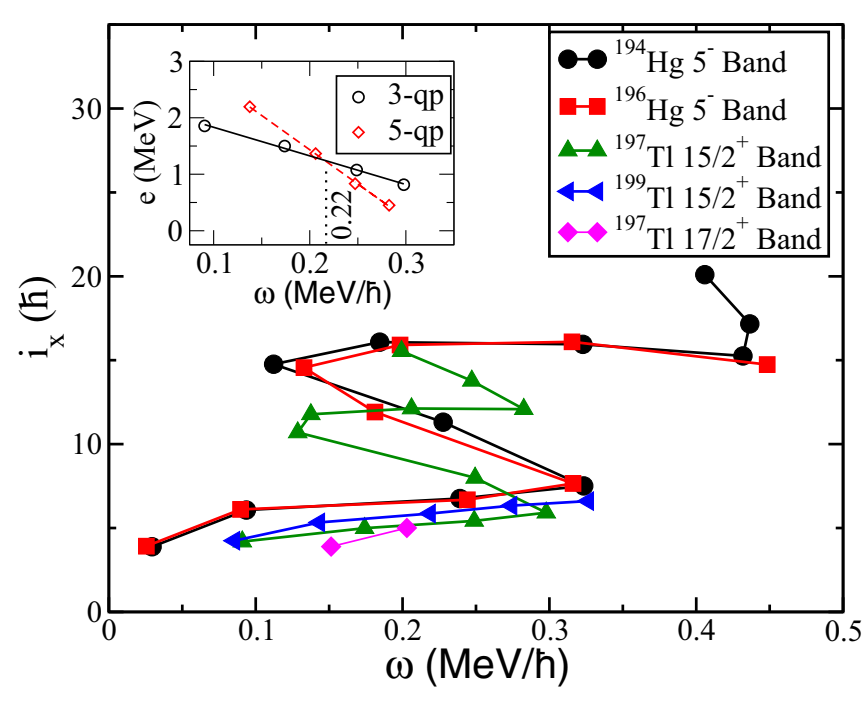

FIG. 13. Aligned angular momentum $i_{x}$ as a function of rotational frequency $(\omega)$ for the bands $D$ and $E$, based on the $15 / 2^{+}$and $17 / 2^{+}$states, respectively, in ${ }^{197} \mathrm{Tl}$. Same quantities for the 3-qp band in the neighboring isotope ${ }^{199} \mathrm{Tl}$ and for the $5^{-}$bands in ${ }^{194,196} \mathrm{Hg}$ are also shown. (Inset) the experimental Routhian (e) vs. rotational frequency for the band $D$ in ${ }^{197} \mathrm{Tl}$. The solid and the dashed lines are the linear fits to the 3-qp and the 5-qp parts of the band $D$. The crossing frequency is indicated by the dotted line.

alignment of an additional pair of neutrons in the low- $\Omega$ components of the $i_{13 / 2}$ orbital located near the Fermi level for oblate deformation [39]. Therefore, a configuration of $\pi h_{9 / 2} \otimes v i_{13 / 2}^{-3}(p f)^{-1}$ is proposed for this 5-qp band in ${ }^{197} \mathrm{Tl}$. The gain in aligned angular momentum $\left(i_{x}\right)$ in Fig. 13 is consistent with this assignment. The experimental Routhians (e) for the 3-qp and 5-qp parts of band $D$ are shown in the inset of Fig. 13. The crossing frequency has been identified as $\hbar \omega=0.22 \mathrm{MeV}$ from this plot. This crossing frequency agrees well with $\hbar \omega=0.23 \mathrm{MeV}$ reported for the $5^{-}$band in ${ }^{196} \mathrm{Hg}$ [39].

A doubly degenerate 5-qp negative parity band at slightly higher excitation energy ( $\approx 4 \mathrm{MeV})$ was observed in ${ }^{195} \mathrm{Tl}[21]$ with the same neutron configuration as in the 5-qp band in ${ }^{197} \mathrm{Tl}$ but the proton in $i_{13 / 2}$ orbital. The total Routhian surface (TRS) calculations suggested a triaxial shape for this band and was interpreted as the evidence for chiral band. However, in case of ${ }^{197} \mathrm{Tl}$, no such partner band has been observed and it is interesting to note that $E 2$ crossover transitions are not observed in this band. Therefore, this 5-qp, positive parity band in ${ }^{197} \mathrm{Tl}$ seems to be different in nature compared to both the 3-qp part of this band in ${ }^{197} \mathrm{Tl}$ and also the 5-qp negative parity band in ${ }^{195} \mathrm{Tl}$.

The bands $B$ and the 5-qp part of the band $D$, have the particle-hole configurations with proton particle and neutron holes both in the high- $j$ orbitals. This is a favorable condition for the generation of angular momentum by shears mechanism and to observe magnetic rotational (MR) bands in nuclei that has small quadrupole deformation [42,43]. Such MR bands have been observed in several isotopes of $\mathrm{Pb}, \mathrm{Tl}$, and $\mathrm{Bi}$ nuclei in the $A \approx 190$ mass region [18,44,45]. The level energies (with respect to the band head energy) of the 

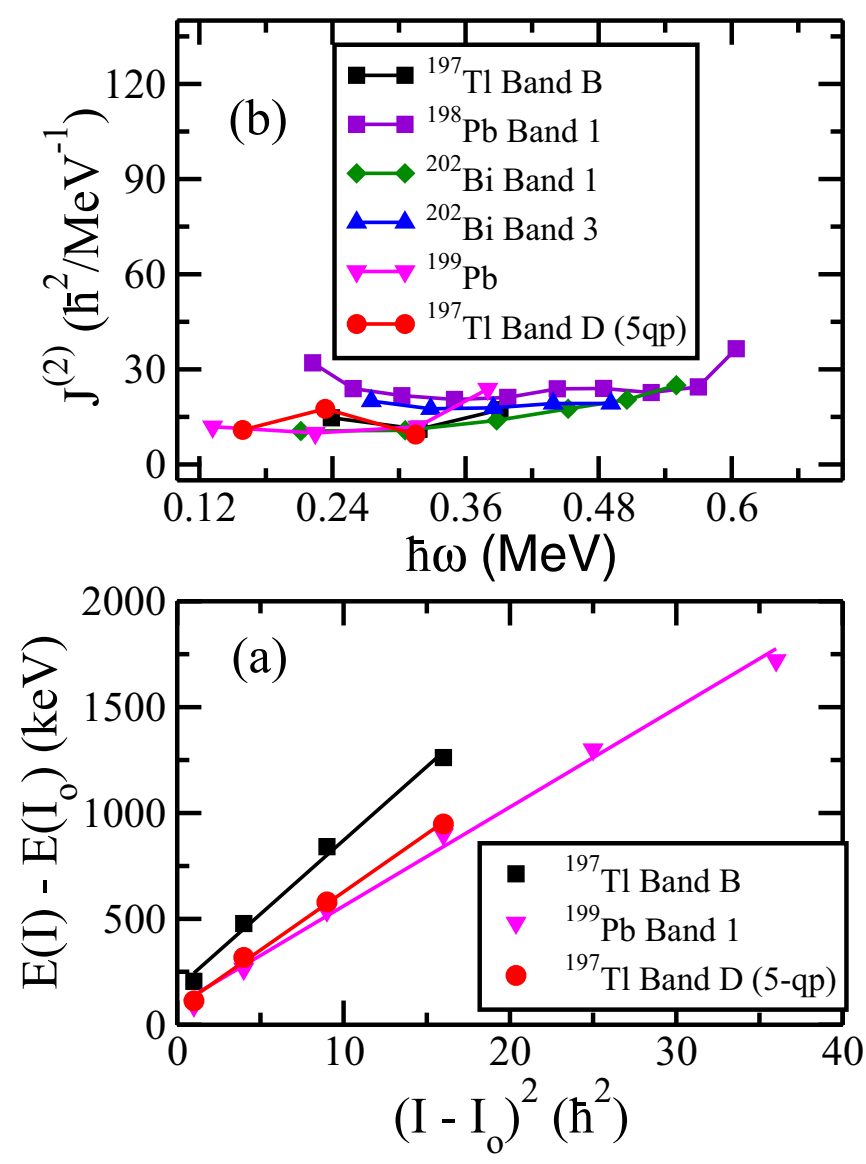

FIG. 14. (a) Level energy $E(I)$ with respect to the band head energy $\left(E_{\circ}\right)$ as a function of the square of spin difference and (b) dynamic moment of inertia $\left(J^{(2)}\right)$ as a function of rotational frequency $(\hbar \omega)$ for the band $B$ in ${ }^{197} \mathrm{Tl}$ and some other MR bands in the neighboring nuclei. The solid lines in (a) are the linear fits to the respective data points (symbol). Data for ${ }^{199} \mathrm{~Pb},{ }^{198} \mathrm{~Pb}$, and ${ }^{202} \mathrm{Bi}$ are taken from Refs. [44,46,47], respectively.

bands $B$ and $D$ in ${ }^{197} \mathrm{Tl}$ are shown in Fig. 14(a) as a function of $\left(I-I_{\circ}\right)^{2}$, where $I$ and $I_{\circ}$ are the level spin and the band head spin, respectively. It shows a straight line behavior as expected for an MR band and similar to a known MR band in ${ }^{199} \mathrm{~Pb}$. The dynamic moment of inertia, $J^{(2)}$ are also plotted for these bands in Fig. 14(b) and are compared with the other MR bands reported in this region. Again the $J^{(2)}$ values for bands $B$ and $D$ in ${ }^{197} \mathrm{Tl}$ have been found to be greater than or $\approx 10 \hbar^{2} \mathrm{MeV}^{-1}$ as expected for an MR band and compares well with the neighboring known MR bands. We have also estimated the experimental $B(M 1) / B(E 2)$ ratios from the measured $\gamma$ ray intensities of the $\Delta I=2$ and $\Delta I=1$ transitions. We have obtained $B(M 1) / B(E 2)=10.4(19)$, 8.4(22), and 6.3(5) for the $29 / 2^{+}, 31 / 2^{+}$, and $33 / 2^{+}$levels of the band $B$. Considering that the shape of the nucleus [and hence, the $B(E 2)$ value] remains same throughout the band, a decrease in the value of $B(M 1)$ is evident for this band. Although we do not have the measured lifetime data, but all other conditions clearly suggest the MR nature.

This gives an interesting aspect of the odd- $A \mathrm{Tl}$ isotopes. While a pair of doubly degenerate bands, an indication of chiral bands, have been observed for the $N=114$ isotope ${ }^{195} \mathrm{Tl}$, a pair of MR bands has been found in the $N=116$ isotope ${ }^{197} \mathrm{Tl}$. Therefore, as the neutron Fermi level increases towards the top of the $i_{13 / 2}$ orbital and near the negative parity $(f, p)$ orbitals, the angular momentum vectors seem to move from an aplanar configuration to a planar one. It would be interesting to study the nature of the 5-qp band in ${ }^{199} \mathrm{Tl}$, information on which is limited below the band crossing region [14]. Similar transition from an aplanar to a planar configuration has also been observed in Cs isotopes in $A \approx 130$ mass region [48].

The band $E$ and $F$ are similar to the one reported earlier [13] with tentative spin assignments for the states. This band has been modified in the present work based on the interband coincidence relation and firm assignment of spin and parity has been done. The excitation energy of the band $E$ is very similar to the lower part of band $D$ and therefore, seems to be 3 -qp in nature. This band decays to the $15 / 2^{+}$band head of the band $D$ and has been interpreted as the multiplet of the band $D$, which has a configuration of $\pi h_{9 / 2} \otimes v i_{13 / 2}(f p)$. The plot of aligned angular momenta, $i_{x}$ for the band $E$ in Fig. 13 shows very similar values as those of the band $D$ at the beginning of the band. This behavior is expected for a multiplet with same configuration. Band structure, similar to that of band $E$ has been observed in ${ }^{199} \mathrm{Tl}$ [14] also.

The bands $B$ and $D$ have been further investigated in the framework of a semiclassical model of magnetic rotation [49] to get an idea about particle-hole interaction. This model is based on the coupling between proton blade $\left(j_{\pi}\right)$ and neutron blade $\left(j_{v}\right)$ to produce the total angular momentum $(I)$. The shears angle $\theta$ that corresponds to a given state in the band can be derived using the semiclassical expression [49],

$$
\cos \theta=\frac{I(I+1)-j_{\pi}\left(j_{\pi}+1\right)-j_{\nu}\left(j_{\nu}+1\right)}{2 \sqrt{j_{\pi}\left(j_{\pi}+1\right) j_{\nu}\left(j_{\nu}+1\right)}},
$$

where $I$ is the total angular momentum. The values of $j_{\pi}$ and $j_{v}$ are $5.5 \hbar$ and $11 \hbar$, respectively, for the proposed 3-qp configuration $\pi i_{13 / 2} \otimes v i_{13 / 2}^{-2}$ for the band $B$. In case of the 5-qp part of the band $D, j_{\pi}=4.5$ and $j_{\nu}=13$ are considered. The band head spins of $12.5 \hbar$ and $13.5 \hbar$ are well reproduced for the 3-qp and the 5-qp MR bands, respectively, by assuming perpendicular coupling between $j_{\pi}$ and $j_{\nu}$. The maximum spins given by the alignment of proton $\left(j_{\pi}\right)$ and neutron $\left(j_{v}\right)$ angular momenta along the total angular momentum axis would be $16.5 \hbar$ and $17.5 \hbar$ for the 3-qp and 5-qp bands, respectively. Irregular behavior in the level spacing has been observed above $33 / 2^{+}$and $37 / 2^{+}$levels for the 3-qp and the 5-qp bands, respectively, at which point band crossings seem to have taken place in these two bands. Therefore, the maximum spin values obtained from the assigned configuration are also consistent with the highest spins observed for the two MR bands.

According to the prescription of Macchiavelli et al. [49] in the case of shears mechanism, the neutron and proton angular momenta are coupled to spin $I$ and interact via a term of the form $V_{2} P_{2}(\cos \theta)$. The energy along the band is given only by the change in potential energy due to the angular momentum coupling, and accordingly, the excitation energies of the states in the MR bands, with respect to band head energy, can be 

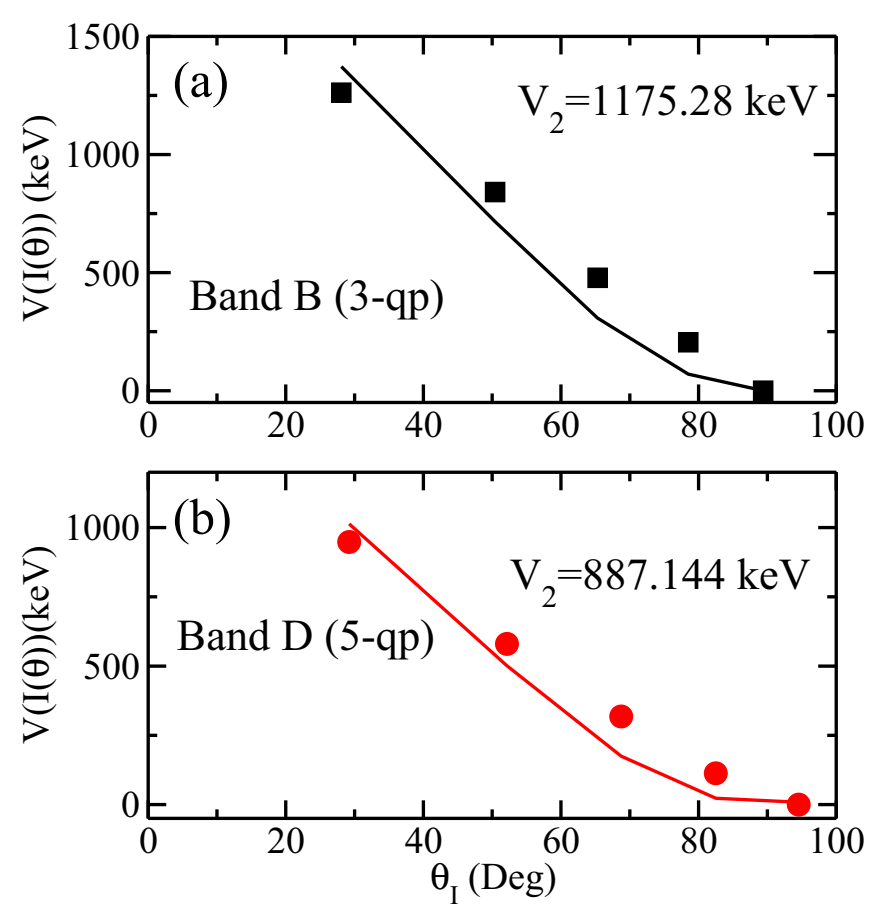

FIG. 15. Plot of $V[I(\theta)]$ as a function of shears angle $\theta$ for (a) the band $B$ and (b) the band $D$ in ${ }^{197} \mathrm{Tl}$. The solid lines are the fits to the data points from which the effective interaction $V_{2}$ between the proton and neutron angular momentum vectors has been obtained.

written as

$$
V[I(\theta)]=E_{I}-E_{b}=(3 / 2) V_{2} \cos ^{2}\left(\theta_{I}\right),
$$

where $E_{I}$ and $E_{b}$ are the level energy corresponding to the angular momentum $I$ and the band head energy, $\theta_{I}$ is the shears angle related to the angular momentum $I$, and $V_{2}$ is the total interaction strength between the proton and the neutron blades. The experimental $V[I(\theta)]$ and $\theta_{I}$ have been extracted and are plotted in Fig. 15 for the two MR bands in ${ }^{197} \mathrm{Tl}$. The experimental data points are fitted by using Eq. (4) to extract the value of the interaction strength $V_{2}$. The fitted values of $V_{2}$ are also shown in the figure. We have obtained $V_{2}=1175 \mathrm{keV}$ for the 3-qp MR band and $V_{2}=887 \mathrm{keV}$ for the 5-qp MR band. These lead to the interaction strength per particle-hole pair as $V_{2}^{p h}=587.5 \mathrm{keV}$ and $221.8 \mathrm{keV}$, respectively, for the 3-qp and 5-qp bands. The value of $V_{2}^{p h}$ for the 3-qp band is similar to the values reported in the $\mathrm{Pb}$ region [50], but it is some what less for the 5-qp band. It is to be noted that the 5-qp configuration also includes low- $j$ negative parity orbitals, hence, it seems that not all of the neutron holes are taking part in the shears mechanism and the interaction strength may not be equally divided among all the particle-hole pairs.

\section{THEORETICAL CALCULATIONS}

\section{A. TRS calculations}

In order to understand the underlying shapes and the effect of the different proton and the neutron orbitals, near the Fermi

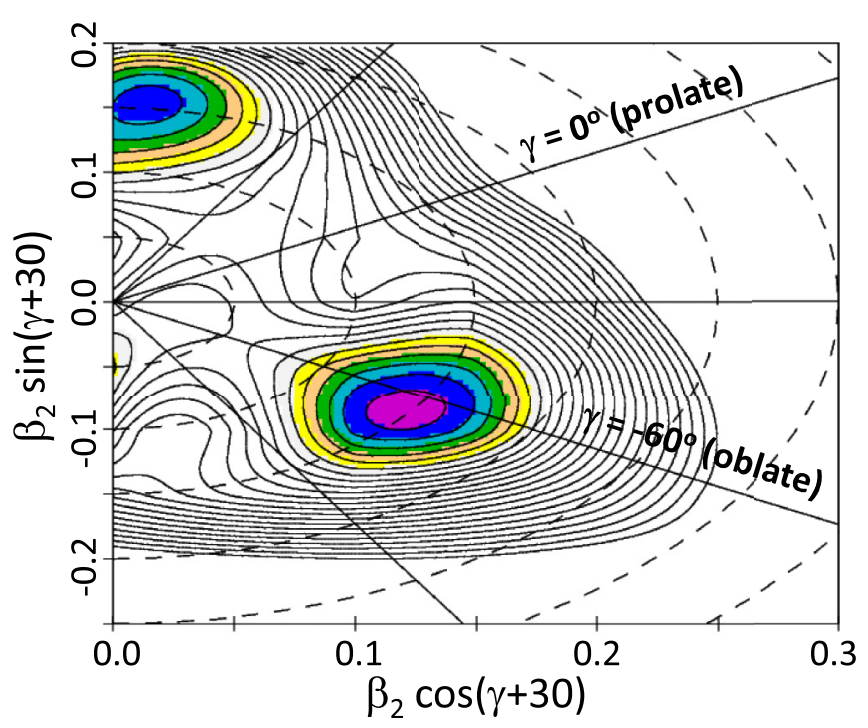

FIG. 16. The total Routhian surfaces calculated for ${ }^{197} \mathrm{Tl}$ for the configuration of $\pi h_{9 / 2} \otimes v i_{13 / 2}^{-2}$, that is after the band crossing in band $C$. The contours are $250 \mathrm{keV}$ apart.

levels, total Routhian surface (TRS) calculations have been performed. The formalism of Nazarewicz et al., as depicted in Refs. [51,52], has been used and the technical procedure has been given in Ref. [53] (and references therein). The TRS calculations are performed in the $\beta_{2}$ and $\gamma\left(\gamma=0^{\circ}=\right.$ prolate and $\gamma=-60^{\circ}=$ oblate) mesh points and minimization on $\beta_{4}$ was done for each value of $\beta_{2}$ and $\gamma$. The TRSs have been calculated for different configurations and at several rotational frequencies $(\hbar \omega)$.

The TRS for the 1-qp configurations of $\pi h_{9 / 2}$ and $\pi i_{13 / 2}$ in ${ }^{197} \mathrm{Tl}$ were earlier calculated in Ref. [13] using the same procedure. It showed oblate shapes for the $\pi h_{9 / 2}$ configuration and nearly spherical shape for the $\pi i_{13 / 2}$ configuration.

The calculated TRSs for the configuration corresponding to the band $C$ in ${ }^{197} \mathrm{Tl}$ after the band crossing is shown in Fig. 16. It shows the minimum at near oblate deformation with $\beta_{2} \approx 0.15$ and $\gamma \approx-62^{\circ}$ that is similar to the 1-qp $\pi h_{9 / 2}$ configuration. The TRSs for the 3-qp, $\pi i_{13 / 2} \otimes v i_{13 / 2}^{-2}$ configuration corresponding to the band $B$ in ${ }^{197} \mathrm{Tl}$ are shown in Fig. 17. The minimum of the TRS for this configuration indicates a near spherical shape with $\beta_{2} \approx 0.08$ and $\gamma \approx-72^{\circ}$. Similar shape was obtained for the 1-qp $\pi i_{13 / 2}$ configuration in ${ }^{197} \mathrm{Tl}$ as well [13].

The TRSs for the lower part (3-qp) and the upper (5-qp) part of the band $D$ are shown in Fig. 18 and Fig. 19, respectively. An oblate shape is predicted for the 3-qp configuration from the minimum of the TRSs at $\beta_{2} \approx 0.14$ and $\gamma \approx-66^{\circ}$. On the other hand, the TRSs for the 5-qp configuration of $\pi h_{9 / 2} \otimes \nu i_{13 / 2}^{-3}(f p)^{-1}$ corresponding to the upper part of the band $D$ in ${ }^{197} \mathrm{Tl}$ show a minimum at a very low deformation with $\beta_{2} \approx 0.05$ and $\gamma \approx-30^{\circ}$. The near-spherical shape suggested for the band $B$ and the 5-qp part of the band $D$ from the TRS calculations are consistent with the observed MR nature of these bands.

It is interesting to note that a second minimum, about $400 \mathrm{keV}$ above the first one, appears in the TRS plot of the 


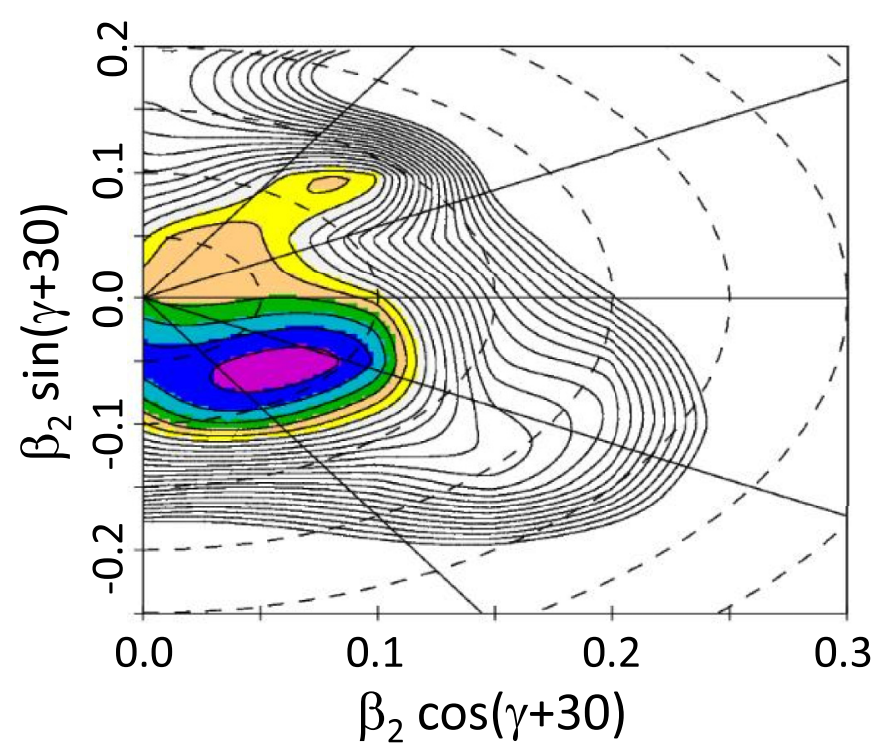

FIG. 17. Same as Fig. 16 but for the 3-qp configuration of $\pi i_{13 / 2} \otimes v i_{13 / 2}^{-2}$ corresponding to the band $B$ in ${ }^{197} \mathrm{Tl}$.

3-qp configuration of band $D$ (Fig. 18). The observation of the band $E$ (Fig. 4) at about the similar excitation energy relative to band $D$ provides an impression that the band $E$ may correspond to this second minimum.

\section{B. SPAC calculations}

Recently, the shears mechanism with the principal axis cranking (SPAC) model [54-58] has been identified as a powerful tool to explore the intrinsic character, quasiparticle configurations, and contribution of (deformed) core rotation in shears sequences. The two sequences of dipole bands, observed in the present work and denoted by the band $B$ and

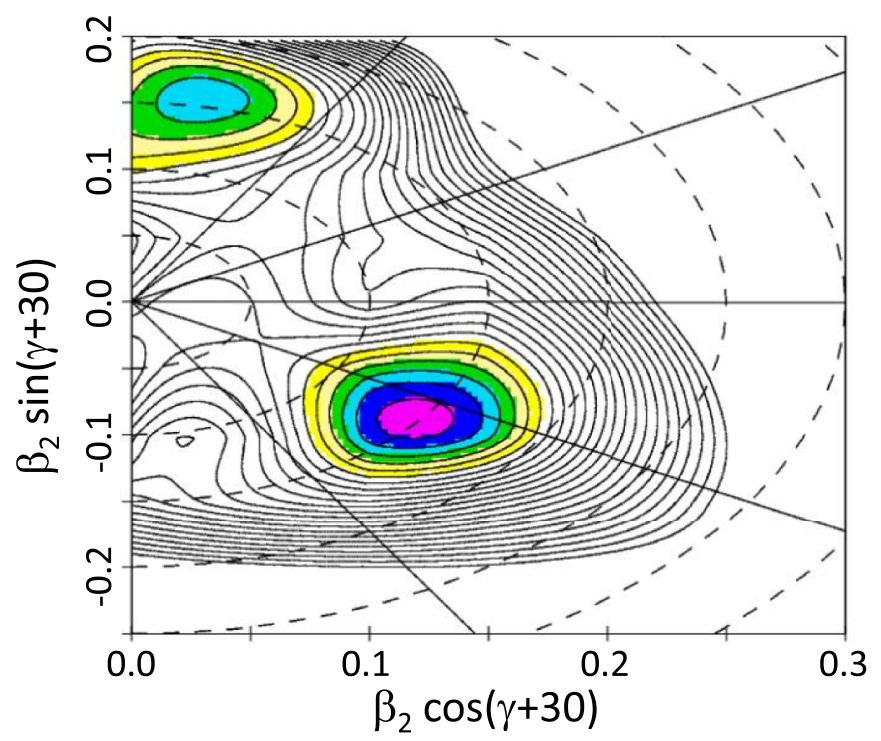

FIG. 18. Same as Fig. 16 but for the 3-qp configuration of $\pi h_{9 / 2} \otimes v i_{13 / 2}^{-1}(f p)^{-1}$ corresponding to the lower part of band $D$ in ${ }^{197} \mathrm{Tl}$.

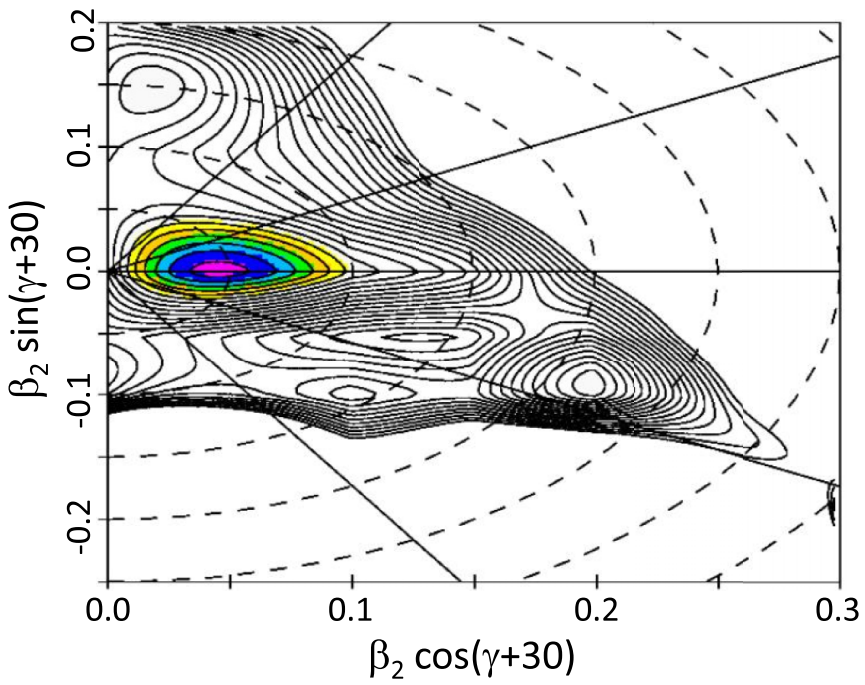

FIG. 19. Same as Fig. 16 but for the 5-qp configuration of $\pi h_{9 / 2} \otimes v i_{13 / 2}^{-3}(f p)^{-1}$ corresponding to the upper part of the band $D$ in ${ }^{197} \mathrm{Tl}$.

the upper part of the band $D$, have been identified as shears bands in ${ }^{197} \mathrm{Tl}$ and are based on the $25 / 2^{+}$and the $27 / 2^{+}$ states, respectively. We have employed SPAC model in order to understand the shears mechanisms in these two bands in ${ }^{197} \mathrm{Tl}$.

In the SPAC model, the shears angular momentum $\left(\overrightarrow{j_{s h}}\right)$, produced by the holes and the particles angular momenta, is coupled with the collective core angular momentum vector $\vec{R}$ to generate the total spin of the observed state $(I)$. The total energy of an excited state $E(I)$ can be expressed as,

$$
E(I)=E(\text { core })+E(\text { shears })+\text { const. }
$$

Here,

$$
E(\text { core })=\frac{R^{2}\left(I, \theta_{1}, \theta_{2}\right)}{2 J(I)}
$$

is the energy due to rotation of the core and

$$
E(\text { shears })=V_{2} P_{2}\left[\cos \left(\theta_{1}-\theta_{2}\right)\right]
$$

is the quasiparticle energy due to the interaction between the shear angular momenta $\overrightarrow{j_{1}}$ and $\overrightarrow{j_{2}}$. Here, $\theta_{1}$ and $\theta_{2}$ are the angles of $\overrightarrow{j_{1}}$ and $\overrightarrow{j_{2}}$ with respect to the rotational axis directed towards $\vec{R}$, respectively. Generally, for each value of $I, \theta_{1}$ and $\theta_{2}$ can be found from the energy minimization condition,

$$
\frac{\partial^{2} E\left(I, \theta_{1}, \theta_{2}\right)}{\partial \theta_{1} \partial \theta_{2}}=0 .
$$

For normal initial alignment [55], the two-dimensional energy minimization can be replaced by one-dimensional condition,

$$
\frac{\partial E\left(I, \theta_{1}\right)}{\partial \theta_{1}}=0
$$

when the direction of $\overrightarrow{j_{2}}$ is set along the rotational axis, which is then used to obtain $\theta_{1}$ for the excited state with 

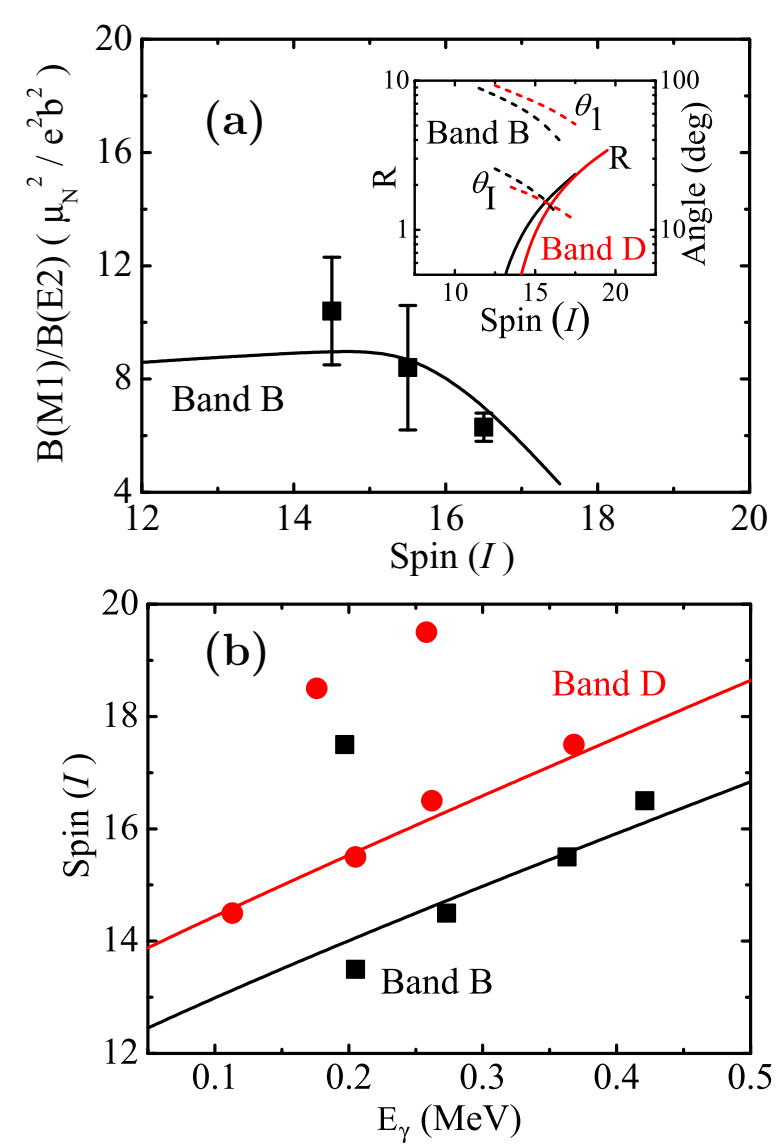

FIG. 20. Comparison of the experimental results for the dipole bands $B$ (represented by the black filled squares) and the upper part of $D$ (represented by the red filled solid circles) in ${ }^{197} \mathrm{Tl}$ with the SPAC model (solid black and red lines for the bands $B$ and upper part of $D$, respectively). The $B(M 1) / B(E 2)$ transition strengths against spin $(I)$ have been depicted in (a) and the variation of spin $(I)$ with the level energy difference $\left(E_{\gamma}\right)$ is shown in (b). The variation of $R$ and $\theta_{1}$ with spin $(I)$ are shown in the inset of (a).

angular momentum $I$. In the subsequent procedure, the level energy difference $\left(E_{\gamma}\right)$ and the transition strengths, $B(M 1)$ and $B(E 2)$, of the state of interest are calculated for this minimized values of $\theta_{1}$ [55,57].

The SPAC model calculations have been carried out with the configurations $\pi i_{13 / 2} \otimes \nu i_{13 / 2}^{-2}$ and $\pi h_{9 / 2} \otimes \nu i_{13 / 2}^{-3}(f p)^{-1}$ for the bands $B$ and the 5-qp part of $D$, respectively, in ${ }^{197} \mathrm{Tl}$. The energy has been minimized for the dipole bands assuming the above configurations and normal initial alignment. The calculations have been performed assuming unstretched condition of the angular momenta with $j_{1}=5.5 \hbar, j_{2}=10 \hbar, V_{2}$ $=0.90-\mathrm{MeV}, g_{1}=-0.15$ and $g_{2}=+1.11$ for band $\mathrm{B}$ and $j_{1}=4.5 \hbar, j_{2}=12 \hbar$, and $V_{2}=+0.85-\mathrm{MeV}$ for the upper part of $D$ [54,57]. The quasiparticle and collective quadrupole moments for the band $B$ have been adopted as $Q_{\text {eff }}=3.5 \mathrm{eb}$ and $Q_{\text {coll }}=1.5 e b$, respectively, to reproduce the experimental $B(M 1) / B(E 2)$ values. Under these assumptions the energy levels and spin $(I)$ values for the dipole bands were well reproduced. The experimental ratio of the $B(M 1) / B(E 2)$ values vs. spin $(I)$ of the states and the $I$ vs. the level energy difference $\left(E_{\gamma}\right)$ are plotted in Figs. 20(a) and 20(b) along with the calculated values from the SPAC model. For initial normal alignment, the spin dependence of $\theta_{1}, \theta_{I}$, and $R$ are also shown in the inset of Fig. 20(a).

The experimental values have been found to be reproduced well for both the bands, assuming that the particle pair is initially not fully stretched. The successful interpretation of the experimental results in the framework of the SPAC model indicates that the dipole band $B$ and upper part of the band $D$ in ${ }^{197} \mathrm{Tl}$ have been generated by the shears mechanism.

\section{SUMMARY}

The excited states in ${ }^{197} \mathrm{Tl}$ have been studied by $\gamma$-ray spectroscopic technique. The reaction ${ }^{197} \mathrm{Au}\left({ }^{4} \mathrm{He}, 4 \mathrm{n}\right){ }^{197} \mathrm{Tl}$ at $50 \mathrm{MeV}$ of beam energy from the K-130 cyclotron at VECC, Kolkata was used to populate the states and the VENUS array with six Compton-suppressed clover HPGe detectors were used to detect the $\gamma$ rays. An improved level scheme of ${ }^{197} \mathrm{Tl}$ with the placement of 28 new $\gamma$-ray transitions has been proposed. The analysis was based on the $\gamma-\gamma$ coincidence relation, DCO ratio, and polarization asymmetry ratio measurements. The new level scheme includes several new band structures. The known oblate bands based on 1-qp $\pi h_{9 / 2}$ and 3-qp $\pi h_{9 / 2} \otimes v i_{13 / 2}^{-1}(f p)^{-1}$ configurations have been extended to observe the $i_{13 / 2}$ neutron alignments. Two new band structures based on a 3-qp (band $B$ ) and a 5-qp (band $D$, upper part) configurations have been identified for the first time in this work which were interpreted as the MR bands. The level ordering and the placement of the $\gamma$-ray transitions of a previously reported band structure, based on the $17 / 2^{+}$state have been rearranged with the placement of a few additional $\gamma$ rays (bands $E$ and $F$ ).

Theoretical calculations have been performed in the framework of the cranking model using the Saxon-Woods potential and BCS pairing. The total Routhian surfaces for the different configurations of the observed band structures in ${ }^{197} \mathrm{Tl}$ have been calculated within this model. The calculations predict that the 1-qp $\pi h_{9 / 2}$ band retains its oblate deformation even after the neutron alignment. However, the calculations suggest a change in shape from an oblate one for the 3-qp band with $\pi h_{9 / 2} \otimes v i_{13 / 2}^{-1}(f p)^{-1}$ configuration to a near spherical one at higher spin after the neutron alignment that is for the $\pi h_{9 / 2} \otimes v i_{13 / 2}^{-3}(f p)^{-1}$ configuration. The near-spherical shape is consistent with the observation of the MR band for this 5-qp configuration. Similar near-spherical shape is obtained in the TRS calculations for the other MR band in this nucleus with 3 -qp configuration of $\pi i_{13 / 2} \otimes \nu i_{13 / 2}^{-2}$. The SPAC model calculations reproduced the experimental observables well and supports the shears mechanism involved in the generation of angular momentum in the bands $B$ and the upper part of $D$.

It is interesting that no evidence of chiral doublet bands has been observed in ${ }^{197} \mathrm{Tl}$ in the present work, unlike the two pairs of doublet band structures reported in ${ }^{195} \mathrm{Tl}$ for 3 -qp and 5-qp configurations. Instead, MR bands have been observed for the 3-qp and 5-qp configurations in ${ }^{197} \mathrm{Tl}$. It may also be noted that neither MR nor chiral bands have been observed in the heavier isotope from ${ }^{199} \mathrm{Tl}$ onwards. It gives an indication 
that perhaps the neutron number $N \approx 116$ forms a boundary for the observation of the MR and chiral bands in odd- $A \mathrm{Tl}$ isotopes. The importance of $N \approx 116$ may be attributed to the fact that the neutron Fermi level goes beyond the high- $j i_{13 / 2}$ orbital above this neutron number. More experimental and theoretical studies are, therefore, warranted in order to fully understand the behaviour of the Tl nuclei as the neutron Fermi level approaches $N=126$.

\section{ACKNOWLEDGMENTS}

The authors thank the VECC cyclotron operators for good quality of $\alpha$ beam throughout the experiment. We also acknowledge the efforts of all who has contributed in the setting up of the VENUS array. One of the authors (H.P.) is grateful for the support from the Ramanujan Fellowship Research Grant under SERB-DST Grant No. SB/S2/RJN-031/2016.
[1] R. M. Diamond et al., Nucl. Phys. A 45, 632 (1963).

[2] V. T. Gritsyna et al., Nucl. Phys. A 61, 129 (1965).

[3] J. O. Newton et al., Nucl. Phys. A 148, 593 (1970).

[4] W. Reviol et al., Phys. Rev. C 61, 044310 (2000).

[5] P. M. Raddon et al., Phys. Rev. C 70, 064308 (2004).

[6] G. J. Lane et al., Phys. Lett. B 324, 14 (1994).

[7] G. J. Lane et al., Nucl. Phys. A 586, 316 (1995).

[8] M.-G. Porquet et al., Phys. Rev. C 44, 2445 (1991).

[9] S. K. Chamoli, P. Joshi, R. Kumar, R. P. Singh, S. Muralithar, R. K. Bhowmik, and I. M. Govil, Phys. Rev. C 75, 054323 (2007).

[10] W. Reviol et al., Phys. Scr. T56, 167 (1995).

[11] W. Reviol et al., Nucl. Phys. A 548, 331 (1992).

[12] R. M. Lieder et al., Nucl. Phys. A 299, 255 (1978).

[13] H. Pai et al., Phys. Rev. C 88, 064302 (2013).

[14] C. B. Li et al., Phys. Rev. C 97, 034331 (2018).

[15] S. Bhattacharya et al., Phys. Rev. C 98, 044311 (2018).

[16] M. G. Slocombe et al., Nucl. Phys. A 275, 166 (1978).

[17] S. Das Gupta, S. Bhattacharyya, H. Pai, G. Mukherjee, S. Bhattacharya, R. Palit, A. Shrivastava, A. Chatterjee, S. Chanda, V. Nanal, S. K. Pandit, S. Saha, J. Sethi, and S. Thakur, Phys. Rev. C 88, 044328 (2013).

[18] H. Pai et al., Phys. Rev. C 85, 064313 (2012).

[19] P. L. Masiteng et al., Phys. Lett. B 719, 83 (2013).

[20] P. L. Masiteng et al., Eur. Phys. J. A 52, 28 (2016).

[21] T. Roy et al., Phys. Lett. B 782, 768 (2018).

[22] E. A. Lawrie et al., Phys. Rev. C 78, 021305(R) (2008).

[23] S. Bhattacharya et al., Phys. Rev. C 95, 014301 (2017).

[24] S. Frauendorf and J. Meng, Nucl. Phys. A 617, 131 (1997).

[25] S. Frauendorf, Rev. Mod. Phys. 73, 463 (2001).

[26] J. Meng, J. Peng, S. Q. Zhang, and S.-G. Zhou, Phys. Rev. C 73, 037303 (2006).

[27] D. Venos et al., Nucl. Phys. A 280, 125 (1977).

[28] S. Bhattacharya et al., DAE-BRNS Symp. Nucl. Phys. 61, 98 (2016).

[29] http://www.tifr.res.in/pell/lamps.html.

[30] D. C. Radford, Nucl. Instrum. Methods Phys. Res. A 361, 297 (1995).
[31] A. Krämer-Flecken et al., Nucl. Instrum. Methods Phys. Res. A 275, 333 (1989).

[32] Md. A. Asgar and G. Mukherjee, Proc. DAE Symp. Nucl. Phys. 62, 104 (2017).

[33] R. Palit, H. C. Jain, P. K. Joshi, S. Nagaraj, B. V. T. Rao, S. S. Chintalpudi, and S. S. Ghugre, PRAMANA-J. Phys. 54, 347 (2000).

[34] H. Xiaolong and Z. Chunmei, Nucl. Data Sheets 104, 283 (2005).

[35] https://www-nds.iaea.org/public/ensdf_pgm/

[36] H. Xiaolong, Nucl. Data Sheets 108, 1093 (2007).

[37] P. H. Regan et al., Nucl. Phys. A 586, 351 (1995).

[38] T. Roy, Ph.D. thesis, Homi Bhabha National Institute, Variable Energy Cyclotron Centre, India, 2017, http:/www.hbni.ac.in/ students/dsp_ths.html?nm=phys/PHYS04201104003.pdf

[39] D. Mehta et al., Z. Phys. A 339, 317 (1991).

[40] H. Helppi, S. K. Saha, P. J. Daly, S. R. Faber, and T. L. Khoo, Phys. Rev. C 28, 1382 (1983).

[41] H. Häbel et al., Nucl. Phys. A 453, 316 (1986).

[42] R. M. Clark and A. O. Macchiavelli, Annu. Rev. Nucl. Part. Sci. 50, 1 (2000).

[43] A. K. Jain et al., Pramana 75, 51 (2010).

[44] G. Baldsiefen et al., Nucl. Phys. A 574, 521 (1994).

[45] H. Pai et al., Phys. Rev. C 90, 064314 (2014).

[46] A. Gärgen et al., Nucl. Phys. A 683, 108 (2001).

[47] R. M. Clark et al., J. Phys. G: Nucl. Part. Phys. 19, L57 (1993).

[48] H. Pai et al., Phys. Rev. C 84, 041301(R) (2011).

[49] A. O. Macchiavelli et al., Phys. Rev. C 57, R1073(R) (1998).

[50] A. O. Macchiavelli, R. M. Clark, M. A. Deleplanque, R. M. Diamond, P. Fallon, I. Y. Lee, F. S. Stephens, and K. Vetter, Phys. Rev. C 58, R621(R) (1998).

[51] W. Nazarewicz et al., Nucl. Phys. A 435, 397 (1985).

[52] W. Nazarewicz et al., Nucl. Phys. A 512, 61 (1990).

[53] G. Mukherjee et al., Nucl. Phys. A 829, 137 (2009).

[54] A. A. Pasternak et al., Eur. Phys. J. A 23, 191 (2005).

[55] E. O. Podsvirova et al., Eur. Phys. J. A 21, 1 (2004).

[56] A. A. Pasternak et al., Eur. Phys. J. A 37, 279 (2008).

[57] S. Rajbanshi et al., Phys. Rev. C 89, 014315 (2014).

[58] S. Rajbanshi et al., Phys. Rev. C 90, 024318 (2014). 